New Theory

ISSN: 2149-1402
37 (2021) 8-25

Journal of New Theory

https://dergipark.org.tr/en/pub/jnt

Open Access

\title{
New Exact Solutions of Conformable Time-Fractional Bad and Good Modified Boussinesq Equations
}

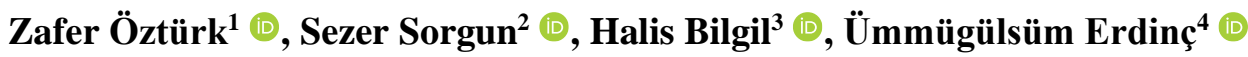

\author{
Article History \\ Received: 03 Nov 2021 \\ Accepted: 22 Dec 2021 \\ Published: 31 Dec 2021 \\ 10.53570/jnt. 1018383 \\ Research Article
}

\begin{abstract}
The new exact solutions of the conformable time-fractional Bad and Good modified Boussinesq equations are obtained using the Exp-function method, which is different from previous literature works. These equations play a significant role in mathematical physics, engineering sciences and applied mathematics. Plentiful exact solutions with arbitrary parameters are effectively obtained by the method. The obtained solutions are shown graphically. It is shown that the Exp-function method provides a simpler but more effective mathematical tool for constructing exact solutions of non-linear evolution equations.
\end{abstract}

Keywords - Conformable time fractional Bad and Good modified Boussinesq equations, conformable fractional derivative, exact solution, Exp-function method

Mathematics Subject Classification (2020) - 35R11, 34A08

\section{Introduction}

Many phenomena in the real world are governed by nonlinear evolution equations (NLEEs). Hence, it is essential to obtain exact solutions to these equations, and many methods have been proposed to get exact solutions. The most known methods are the Exp-function method [1], the tanh-method [2], the homogeneous balance method [3], the trial function method [4], the $\left(\frac{G^{\prime}}{G}\right)$ expansion methods [5], the Kudryashov method [6]. The Exp-function method was used by many researchers to solutions of various NLEEs [7-10]. Also, new exact solutions of the nonlinear evolution equations may be obtained using different methods.

Travelling waves arise naturally in many physical systems, usually described by partial differential equations. Solitary waves, also known as 'solitons', are a particular travelling wave class with some special properties. Solitons can usually propagate over large distances without dispersion due to certain nonlinear effects cancelling out dispersive effects. They also have the additional property that they can interact with other solitons such that they emerge following a collision without changing shape, apart from a small phase change.

\footnotetext{
${ }^{1}$ zaferozturk@aksaray.edu.tr (Corresponding Author); $\quad{ }^{2}$ ssorgun@nevsehir.edu.tr; $\quad{ }^{3}$ halis@aksaray.edu.tr;

${ }^{4}$ ummugulsumerdinc@aksaray.edu.tr

1,2 Department of Mathematics, Nevşehir HacıBektaş Veli University, Nevşehir, Turkey

${ }^{3,4}$ Department of Mathematics, Aksaray University, Aksaray, Turkey
} 
Traditional real problems are defined with integer-order nonlinear evolution equations, characterized by theirs. However, the nonlinear evolution equations with integer-order derivatives are ideal classic events, which are not suitable for describing irregular phenomena. On the other hand, Fractional differential equations have become preferable, despite the difficulty in calculations, since they give more real results than standard integer order nonlinear evolution equations. Difficulties in fractional calculus have begun to be overcome thanks to new fractional derivative definitions and theorems made in recent years. The most popular definitions of the fractional derivative can be listed as the conformable derivative [11], the Caputo derivative [12], Riemann-Liouville derivative [12], Atangana-Baleanu derivative [13]. The new trends in exact solution research are to find new exact solutions and to develop new solution mechanisms.

Lately, Khalil et al. defined a limit-based fractional derivative in 2014 [11], named the conformable fractional derivative. The structure of this new definition of fractional derivative is simpler than that of other popular fractional derivatives.

The definition of conformable fractional derivative is given as follow.

Let $f:[0, \infty] \rightarrow \mathbb{R}$. Then, the conformable fractional derivative of order $\alpha$ is defined by

$$
T_{\alpha}(f(t))=\lim _{\varepsilon \rightarrow 0} \frac{f\left(t+\varepsilon t^{1-\alpha}\right)-f(t)}{\varepsilon}
$$

for all $t>0$ and $\alpha \in(0,1]$. If $f$ is $\alpha$-differentiable in some $(0, \alpha), \alpha>0$ and $\lim _{t \rightarrow 0^{+}} T_{\alpha}(f(t))$ exists, then

$$
\left(T_{\alpha} f\right)(0)=\lim _{t \rightarrow 0^{+}} T_{\alpha}(f(t))
$$

In addition to this definition, it is known that $T_{\alpha}(f(t))=\lim _{\varepsilon \rightarrow 0} \frac{f^{[\alpha-1]}\left(t+\varepsilon t^{[\alpha]-\alpha}\right)-f^{[\alpha-1]}(t)}{\varepsilon}$ such that $\alpha \in$ $(n, n+1]$ and function $f$ is $n$-th order differentiable at a point for $t>0$, where $\lceil$.$\rceil ceil function, \lceil\alpha\rceil$ is the smallest integer no larger than $\alpha$ [11].

This paper applies the Exp-function method to new exact solutions of fractional Bad and Good modified Boussinesq equations with conformable derivative. The rest of this paper is organized as follows: Some useful properties of the conformable fractional derivative and mechanism of the Exp-function method are given in Section 2. Exact solutions of the fractional Bad and Good modified Boussinesq equations are obtained in Section 3. Finally, the conclusions of this paper are given in Section 4.

\section{Preliminary and The Exp-Function Method}

It is well known that most of the events that develop in mathematical, physics and engineering fields can be described by partial differential equations (PDEs). So, partial differential equations are useful tools for mathematical modelling. First, the Exp-function method is defined by $\mathrm{He}$ and $\mathrm{Wu}(2006)$ and applied to various applications by many scientists [14-20]. The exact solution of non-linear partial differential equations is obtained by the Exp-function method. First, the partial differential equation is reduced to the ordinary differential equation and referred to the exact solution using the exponential function method. The Expfunction method is an effective method for solutions of the non-linear evolution equations that emerges in mathematical physics, applied mathematics and engineering applications. The Exp-function method also gives generalized single and periodic solutions of the nonlinear evolution equation. Some solutions of fractional Bad and Good modified Boussinesq equations with the aid of auxiliary equation method are obtained by Durur et al. [21].

We consider a general nonlinear PDE in the form

$$
P=\left(x, y, u, u_{x}, u_{y}, u_{x x}, u_{x y}, u_{y y}, \ldots\right)=0
$$

Let us introduce a complex variable $\xi=k x+w t$ where $k$ and $w$ are constants. We rewrite Equation (3) in the subsequent nonlinear ODE: 


$$
Q\left(u, u^{\prime}, u^{\prime \prime}, u^{\prime \prime \prime}, \ldots\right)=0
$$

where the prime denotes the derivation concerning $\xi$ [4]. According to the Exp-function method, we assume that the solution can be expressed in the form

$$
u(\xi)=\frac{\sum_{n=-c}^{d} a_{n} e^{(n \xi)}}{\sum_{m=-p}^{q} b_{m} e^{(m \xi)}}
$$

where $c, d, p$ and $q$ are positive integers, $a_{n}$ and $b_{m}$ are unknown constants to be observed. Without loss of generality, if we take $d=q=c=p=1$ then Equation (5) can be written as follow,

$$
u(\xi)=\frac{a_{-1} \xi+a_{0}+a_{1} e^{\xi}}{b_{-1} e^{-\xi}+b_{0}+e^{\xi}}
$$

Here, the constant $b_{1}$ is taken as 1 for simplicity. It will arrive us to a set of algebraic equations for the unknowns $a_{0}, a_{1}, a_{-1} ; b_{0}, b_{-1} ; k, w$. Some useful theorems are given by Ebaid [22] for this subject.

Theorem 2.1. Suppose that $u^{(r)}$ and $u^{(\gamma)}$ are respectively the highest order linear term and the highest order nonlinear term of a nonlinear ODE, where $r$ and $\gamma$ are both positive integers. Then the balancing procedure using the Exp-function ansatz; $u(\xi)=\frac{\sum_{n=-c}^{d} a_{n} e^{(n \xi)}}{\sum_{m=-p}^{q} b_{m} e^{(m \xi)}}$ leads to $c=d$ and $p=q, \forall r \geq 1, \forall \gamma \geq 1$ [14].

i) Suppose that $u^{(r)}$ and $u^{(s)} u^{(k)}$ are respectively the highest order linear term and the highest order nonlinear term of a nonlinear ODE, where $r, s$ and $k$ are all positive integers. Then the balancing procedure using the Exp-function ansatz leads to $c=d$ and $p=q, \forall r, s, k \geq 1$

ii) Let $u^{(r)}$ and $\left(u^{(s)}\right)^{\gamma}$ be respectively the highest order linear term and the highest order nonlinear term of a nonlinear ODE, where $r, s$ and $\gamma$ are all positive integers. Then the balancing procedure using the Expfunction ansatz leads to $c=d$ and $p=q, \forall r, s \geq 1, \forall \gamma \geq 2$

iii) Suppose that $u^{(r)}$ and $\left(u^{(s)}\right)^{\gamma} u^{(\lambda)}$ are respectively the highest order linear term and the highest order nonlinear term of a nonlinear ODE, where $r, s, \gamma$ and $\lambda$ are all positive integers. Then the balancing procedure using the Exp-function ansatz leads to $c=d$ and $p=q, \forall r, s, \gamma, \lambda \geq 1$

Theorem 2.2. [11] If $f$ and $g$ functions are $\alpha$-differentiable at a point $t>0$ for $\alpha \in(0,1]$, then

a) $T_{\alpha}(\lambda f(t)+\delta g(t))=\lambda T_{\alpha}(f(t))+\delta T_{\alpha}(g(t))$, for all $\delta, \lambda \in \mathbb{R}$

b) $T_{\alpha}\left(t^{p}\right)=p t^{p-\alpha}$, for all $p \in \mathbb{R}$

c) $T_{\alpha}(c)=0$ for all constant $c$.

d) $T_{\alpha}(f(t) g(t))=f(t) T_{\alpha}(g(t))+g(t) T_{\alpha}(f(t))$

e) $T_{\alpha}\left(\frac{f(t)}{g(t)}\right)=\frac{g(t) T_{\alpha}(f(t))-f(t) T_{\alpha}(g(t))}{(g(t))^{2}}$

f) If the function $f$ is differentiable, $T_{\alpha}(f(t))=t^{1-\alpha} \frac{d f}{d t}$.

In addition, $\alpha$-th order conformable fractional derivatives of some functions are given as,

i) $T_{\alpha}\left(e^{a t}\right)=a t^{1-\alpha} e^{a t}, \forall a \in \mathbb{R}$

ii) $T_{\alpha}(\sin (b t))=b t^{1-\alpha} \cos (b t), \forall b \in \mathbb{R}$

iii) $T_{\alpha}(\cos (c t))=-c t^{1-\alpha} \sin (c t), \forall c \in \mathbb{R}$

iv) $T_{\alpha}\left(\frac{t^{\alpha}}{\alpha}\right)=1$ 

v) $T_{\alpha}\left(\sin \left(\frac{t^{\alpha}}{\alpha}\right)\right)=\cos \left(\frac{t^{\alpha}}{\alpha}\right)$
vi) $T_{\alpha}\left(\cos \left(\frac{t^{\alpha}}{\alpha}\right)\right)=-\sin \left(\frac{t^{\alpha}}{\alpha}\right)$
vii) $T_{\alpha}\left(e^{\frac{t^{\alpha}}{\alpha}}\right)=e^{\frac{t^{\alpha}}{\alpha}}$

\section{Fractional Bad and Good Modified Boussinesq Equations}

The Boussinesq equation is first discovered by Joseph Boussinesq in 1870 [23]. This equation is one of the non-linear partial differential equations in plasma that have applications in many areas, such as ion sound waves, shallow water waves modelling, longitudinal propagation waves in elastic bars, suppressed waves in liquid-gas foam mixtures and the propagation model of these waves. Then, the Boussinesq equation is modified to adapt to deeper water problems and hence there are many new forms of this equation in the literature [21,23].

Time-Fractional Bad Modified Boussinesq Equation is denoted by

$$
D_{t}^{(2 \alpha)} u-D_{x}^{2} u-D_{x}^{4} u-3 D_{x}^{2}\left(u^{2}\right)+3 D_{x}\left(u^{2} D_{x} u\right)=0
$$

Time-Fractional Good Modified Boussinesq Equation is denoted by

$$
D_{t}^{(2 \alpha)} u-D_{x}^{2} u+D_{x}^{4} u-3 D_{x}^{2}\left(u^{2}\right)+3 D_{x}\left(u^{2} D_{x} u\right)=0
$$

We are now ready for solutions of Time-Fractional Bad Modified Boussinesq Equation using the Expfunction method to produce a solution set. In view of the Exp-function method, we assume that the solutions of Equation (7) can be expressed in the form,

$$
u(\xi)=\frac{\sum_{n=-c}^{d} a_{n} e^{(n \xi)}}{\sum_{m=-p}^{q} b_{m} e^{(m \xi)}}
$$

By using Theorem 2. 2. (f) and the travelling wave transformation as follow,

$$
u(x, t)=u(\xi), \xi=x-w \frac{t^{\alpha}}{\alpha}
$$

and therefore, the Equation (7) convert to an ordinary differential equation. Here $a_{n}$ and $b_{m}$ are unknown constants, $w$ denotes the velocity of the wave, and prime denotes the derivative of the functions concerning $\xi$.

By applying the wave transform in (9), the equation in (7) is obtained to the following ordinary differential equation.

$$
\left(-\mathrm{w}^{2}-1\right) u^{\prime \prime}-u^{\imath v}-6 u^{\prime 2}-6 u u^{\prime \prime}+6 u u^{\prime 2}+3 u^{2} u^{\prime \prime}=0
$$

By applying the wave transform in (9), the equation in (8) is obtained to the following ordinary differential equation.

$$
\left(-\mathrm{w}^{2}-1\right) u^{\prime \prime}+u^{l v}-6 u^{\prime 2}-6 u u^{\prime \prime}+6 u u^{\prime 2}+3 u^{2} u^{\prime \prime}=0
$$

Firstly, substitute Equation (6) into Equation (10) and Equation (11), then the unknown parameters are obtained by using Maple Software.

Hence, we obtain all the solutions related to Bad and Good Modified Boussinesq Equations cases. We balance the linear term of the highest order of Equation (10) $u^{l v}$ with the highest order nonlinear term $u^{2} u^{\prime \prime}$, we set $p=c=1$ and $q=d=1$; then the trial solution of Equation (10), reduces to

$$
\frac{-1}{A}\left[C_{4} e^{4 \xi}+C_{3} e^{3 \xi}+C_{2} e^{2 \xi}+C_{1} e^{\xi}+C_{0}+C_{-1} e^{-\xi}+C_{-2} e^{-2 \xi}+C_{-3} e^{-3 \xi}+C_{-4} e^{-4 \xi}\right]=0
$$


and all coefficients must be zero for the solutions of Equation (12). Hence, we get

$$
\begin{aligned}
& A=b_{-1}\left(e^{-\xi}+b_{0}+e^{\xi}\right)^{5} \\
& C_{4}=-2 a_{0}+3 a_{1}^{2} a_{0}-6 a_{0} a_{1}+6 a_{1}^{2} b_{0}-3 a_{1}^{3} b_{0}+2 a_{1} b_{0}-w^{2} a_{0}+w^{2} a_{1} b_{0} ; \\
& C_{3}=-20 a_{-1}+24 a_{1}^{2} b_{-1}+20 a_{1} b_{-1}+12 a_{1}^{2} a_{-1}+9 a_{1}^{3} b_{0}^{2}-6 a_{1}^{2} b_{0}^{2}-12 a_{1}^{3} b_{-1}+12 a_{1} a_{0}^{2} \\
& -24 a_{1} a_{-1}-4 w^{2} a_{-1}-10 a_{1} b_{0}^{2}+10 a_{0} b_{0}+4 w^{2} a_{1} b_{-1}+18 a_{1} b_{0} a_{0}-w^{2} a_{0} b_{0}+w^{2} a_{1} b_{0}^{2} \\
& -21 a_{1}^{2} a_{0} b_{0}-12 a_{0}^{2} \\
& C_{2}=9 a_{0}^{3}+4 w^{2} a_{0} b_{-1}-70 a_{1} b_{-1} b_{0}-12 a_{1}^{2} b_{0} b_{-1}-w^{2} a_{1} b_{0}^{3}-12 a_{1} a_{-1} b_{0}-21 a_{1}^{2} a_{-1} b_{0} \\
& -21 a_{1} b_{0} a_{0}^{2}+12 a_{0} a_{1}^{2} b_{0}^{2}++18 a_{1} a_{0} b_{0}^{2}+78 a_{1} a_{0} b_{-1}-11 w^{2} a_{-1} b_{0}-66 a_{0} a_{1}^{2} b_{-1}-10 a_{0} b_{0}^{2} \\
& +80 a_{0} b_{-1}-54 a_{-1} a_{0}-10 a_{-1} b_{0}-12 a_{1}^{2} b_{0}^{3}++33 a_{1}^{3} b_{0} b_{-1}+w^{2} a_{0} b_{0}^{2}+7 w^{2} a_{1} b_{0} b_{-1} \\
& +10 a_{1} b_{0}^{3}-6 a_{0}^{2} b_{0}+54 a_{1} a_{0} a_{-1} \\
& C_{1}=-w^{2} a_{1} b_{0}^{4}-84 a_{1} a_{0}^{2} b_{-1}+4 w^{2} a_{1} b_{-1}^{2}-24 a_{1}^{2} b_{-1}^{2}+56 a_{1} b_{0}^{2} b_{-1}-6 a_{0} a_{1} b_{0}^{3}-78 a_{-1} a_{0} b_{0} \\
& +3 a_{0}^{2} a_{1} b_{0}^{2}+3 a_{-1} a_{1}^{2} b_{0}^{2}-4 w^{2} a_{-1} b_{-1}+12 a_{-1} a_{1} b_{0}^{2}+72 a_{-1} a_{1} b_{-1}-54 a_{1}^{2} b_{-1} b_{0}^{2}-11 w^{2} a_{-1} b_{0}^{2} \\
& +w^{2} a_{0} b_{0}^{3}-84 a_{1}^{2} a_{-1} b_{-1} \pm 34 a_{0} b_{0} b_{-1}+36 a_{0}^{2} b_{-1}-48 a_{-1}^{2}+2 a_{0} b_{0}^{3}+48 a_{-1} a_{0}^{2}-2 a_{1} b_{0}^{4} \\
& -172 a_{1} b_{-1}^{2}-3 a_{0}^{3} b_{0}+48 a_{1} a_{-1}^{2}+36 a_{1}^{3} b_{-1}^{2}++6 a_{0}^{2} b_{0}^{2}+172 a_{-1} b_{-1}-22 a_{-1} b_{0}^{2} \\
& +51 a_{0} a_{1}^{2} b_{0} b_{-1}+84 a_{0} a_{1} b_{0} b_{-1}+13 w^{2} a_{0} b_{0} b_{-1}-2 w^{2} a_{1} b_{-1} b_{0}^{2}-18 a_{-1} a_{0} a_{1} b_{0} ; \\
& C_{0}=110 a_{-1} b_{-1} b_{0}-90 a_{1}^{2} b_{-1}^{2} b_{0}+15 a_{-1} b_{0} a_{0}^{2}+15 a_{-1} a_{1}^{2} b_{-1} b_{0}+75 a_{0} a_{1}^{2} b_{-1}^{2}+30 a_{0} a_{1} b_{-1}^{2} \\
& -5 w^{2} a_{-1} b_{-1} b_{0}+10 w^{2} a_{0} b_{-1} b_{0}^{2}+15 a_{1} b_{0} a_{0}^{2} b_{-1}+10 w^{2} a_{0} b_{-1}^{2}-10 a_{-1} b_{0}^{3}-220 a_{0} b_{-1}^{2} \\
& +30 a_{-1} a_{0} b_{-1}-5 w^{2} a_{-1} b_{0}^{3}+15 a_{-1}^{2} a_{1} b_{0}+110 a_{1} b_{-1}^{2} b_{0}-10 a_{1} b_{-1} b_{0}^{3}+20 a_{0} b_{-1} b_{0}^{2}-30 a_{0}^{3} b_{-1} \\
& -30 a_{1} a_{0} b_{0}^{2} b_{-1}-30 a_{-1} b_{0}^{2} a_{0}-5 w^{2} a_{1} b_{-1}^{2} b_{0}+60 a_{0}^{2} b_{-1} b_{0}-90 a_{-1}^{2} b_{0}+120 a_{-1} a_{1} b_{-1} b_{0} \\
& -5 w^{2} a_{1} b_{-1} b_{0}^{3}-180 a_{-1} a_{0} a_{1} b_{-1}+75 a_{0} a_{-1}^{2} \\
& C_{-1}=-172 a_{-1} b_{-1}^{2}+172 a_{1} b_{-1}^{3}-24 a_{-1}^{2} b_{-1}+36 a_{0}^{2} b_{-1}^{2}-2 a_{-1} b_{0}^{4}-48 a_{1}^{2} b_{-1}^{3}-54 a_{-1}^{2} b_{0}^{2} \\
& +48 a_{1} a_{0}^{2} b_{-1}^{2}-22 a_{1} b_{-1}^{2} b_{0}^{2}+6 a_{0}^{2} b_{-1} b_{0}^{2}-84 a_{-1} a_{0}^{2} b_{-1}-6 a_{0} a_{-1} b_{0}^{3}-34 a_{0} b_{-1}^{2} b_{0} \\
& +48 a_{-1} a_{1}^{2} b_{-1}^{2}+3 a_{-1}^{2} b_{0}^{2} a_{1}+3 a_{0}^{2} a_{-1} b_{0}^{2}+4 w^{2} a_{-1} b_{-1}^{2}-3 a_{0}^{3} b_{-1} b_{0}+72 a_{1} a_{-1} b_{-1}^{2}-w^{2} a_{-1} b_{0}^{4} \\
& +51 a_{0} a_{-1}^{2} b_{0}-4 w^{2} a_{1} b_{-1}^{3}+w^{2} a_{0} b_{-1} b_{0}^{3}+12 a_{-1} b_{0}^{2} a_{1} b_{-1}+13 w^{2} a_{0} b_{-1}^{2} b_{0}-2 w^{2} a_{-1} b_{0}^{2} b_{-1} \\
& -11 w^{2} a_{1} b_{0}^{2} b_{-1}^{2}+2 a_{0} b_{-1} b_{0}^{3}-84 a_{-1}^{2} a_{1} b_{-1}+84 a_{-1} a_{0} b_{-1} b_{0}-78 a_{1} b_{-1}^{2} a_{0} b_{0} \\
& -18 a_{-1} a_{0} a_{1} b_{-1} b_{0}+36 a_{-1}^{3}+56 a_{-1} b_{-1} b_{0}^{2} \\
& C_{-2}=9 a_{0}^{3} b_{-1}^{2}-12 a_{-1}^{3} b_{0}^{3}+80 a_{0} b_{-1}^{3}+33 a_{-1}^{3} b_{0}+12 a_{0} a_{-1}^{2} b_{0}^{2}+4 w^{2} a_{0} b_{-1}^{3}+10 a_{-1} b_{-1} b_{0}^{3} \\
& -54 a_{0} a_{1} b_{-1}^{3}-70 a_{-1} b_{-1}^{2} b_{0}-12 a_{-1}^{2} b_{-1} b_{0}-66 a_{-1}^{2} a_{0} b_{-1}+78 a_{-1} a_{0} b_{-1}^{2}-10 a_{1} b_{-1}^{3} b_{0}
\end{aligned}
$$




$$
\begin{aligned}
& -6 a_{0}^{2} b_{-1}^{2} b_{0}-10 a_{0} b_{-1}^{2} b_{0}^{2}+18 a_{-1} b_{0}^{2} a_{0} b_{-1}+w^{2} a_{0} b_{-1}^{2} b_{0}^{2}-12 a_{-1} b_{0} a_{1} b_{-1}^{2}-21 a_{-1} b_{0} a_{0}^{2} b_{-1} \\
& -11 w^{2} a_{1} b_{-1}^{3} b_{0}+54 a_{-1} a_{1} a_{0} b_{-1}^{2}-w^{2} a_{-1} b_{0}^{3} b_{-1}-21 a_{-1}^{2} a_{1} b_{0} b_{-1}+7 w^{2} a_{-1} b_{-1}^{2} b_{0} ; \\
C_{-3}= & 18 a_{-1} b_{0} a_{0} b_{-1}^{2}-21 a_{-1}^{2} a_{0} b_{0} b_{-1}-12 a_{-1}^{3} b_{-1}+24 a_{-1}^{2} b_{-1}^{2}-20 a_{1} b_{-1}^{4}+10 a_{0} b_{-1}^{3} b_{0} \\
& -w^{2} a_{0} b_{-1}^{3} b_{0}+w^{2} a_{-1} b_{-1}^{2} b_{0}^{2}-12 a_{0}^{2} b_{-1}^{3}+9 a_{-1}^{3} b_{0}^{2}+20 a_{-1} b_{-1}^{3}-10 a_{-1} b_{-1}^{2} b_{0}^{2}+4 w^{2} a_{-1} b_{-1}^{3} \\
& +12 a_{-1}^{2} a_{1} b_{-1}^{2}-4 w^{2} a_{1} b_{-1}^{4}-6 a_{-1}^{2} b_{0}^{2} b_{-1}+12 a_{-1} a_{0}^{2} b_{-1}^{2}-24 a_{-1} a_{1} b_{-1}^{3} ; \\
C_{-4}= & w^{2} a_{-1} b_{-1}^{3} b_{0}-2 a_{0} b_{-1}^{4}+6 a_{-1}^{2} b_{-1}^{2} b_{0}+2 a_{-1} b_{-1}^{3} b_{0}-3 a_{-1}^{3} b_{0} b_{-1}+3 a_{-1}^{2} a_{0} b_{-1}^{2}-6 a_{-1} a_{0} b_{-1}^{3} \\
& -w^{2} a_{0} b_{-1}^{4} ;
\end{aligned}
$$

All the coefficients of $e^{n \xi}$ must be zero. Hence, we produce a system of algebraic equations which the Maple can tackle to produce the subsequent cases of solutions:

\section{Case 1:}

$$
a_{0}=a_{1} b_{0}, a_{-1}=a_{1} b_{-1}
$$

Replace these outcomes into Equation (6), we produce a subsequent exact solution,

$$
u(\xi)=a_{1}
$$

where $a_{1}$ is a free parameter.
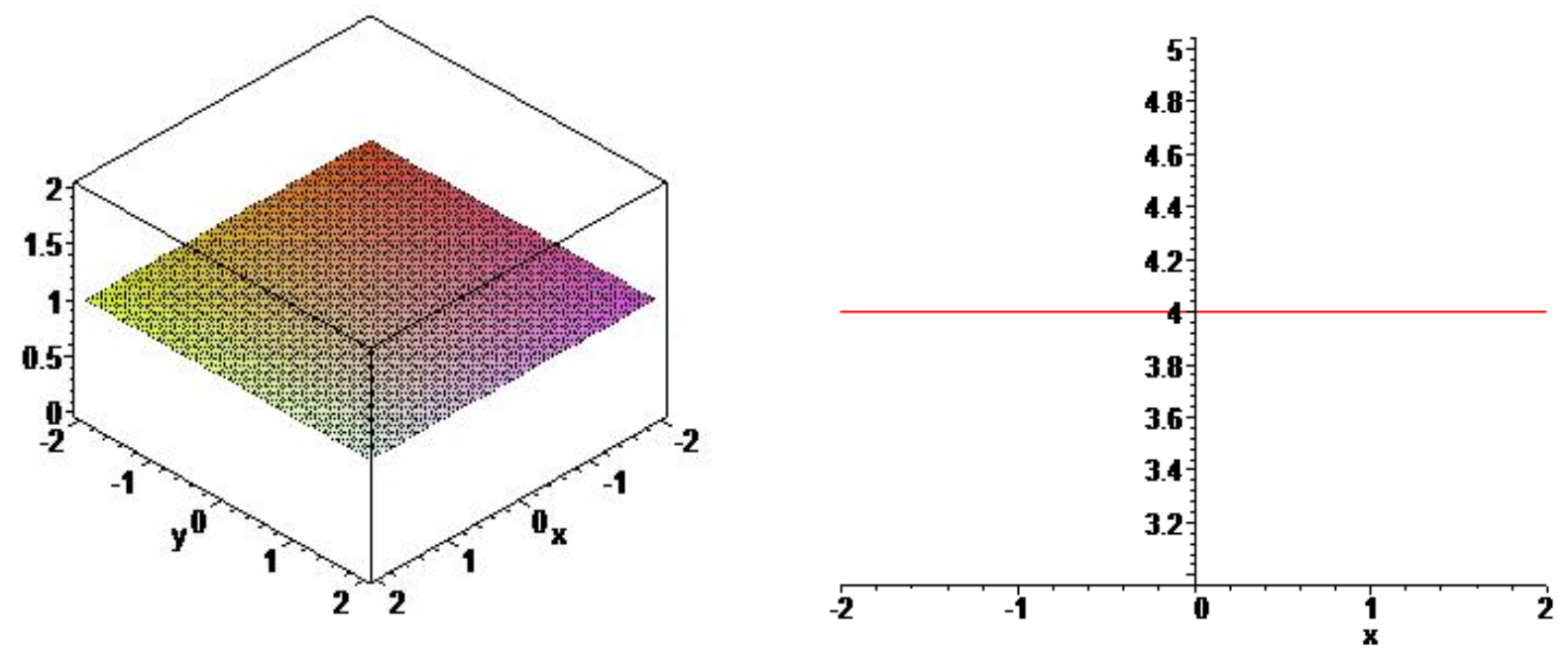

Fig. 1. 3D and 2D plots of travelling wave solutions (Case 1)

The plots indicate the wave solutions for $a_{1}=4$ in Equation (14).

\section{Case 2:}

$$
a_{0}=0, \quad b_{0}=0, a_{1}=1, w=2 \sqrt{2} I, w=-2 \sqrt{2} I, b_{-1}=0
$$

Replace these outcomes into Equation (6), we produce a subsequent exact solution,

$$
u(\xi)=\frac{a_{-1} e^{-\xi}+e^{\xi}}{e^{\xi}}
$$

where $a_{-1}$ is a free parameter. 

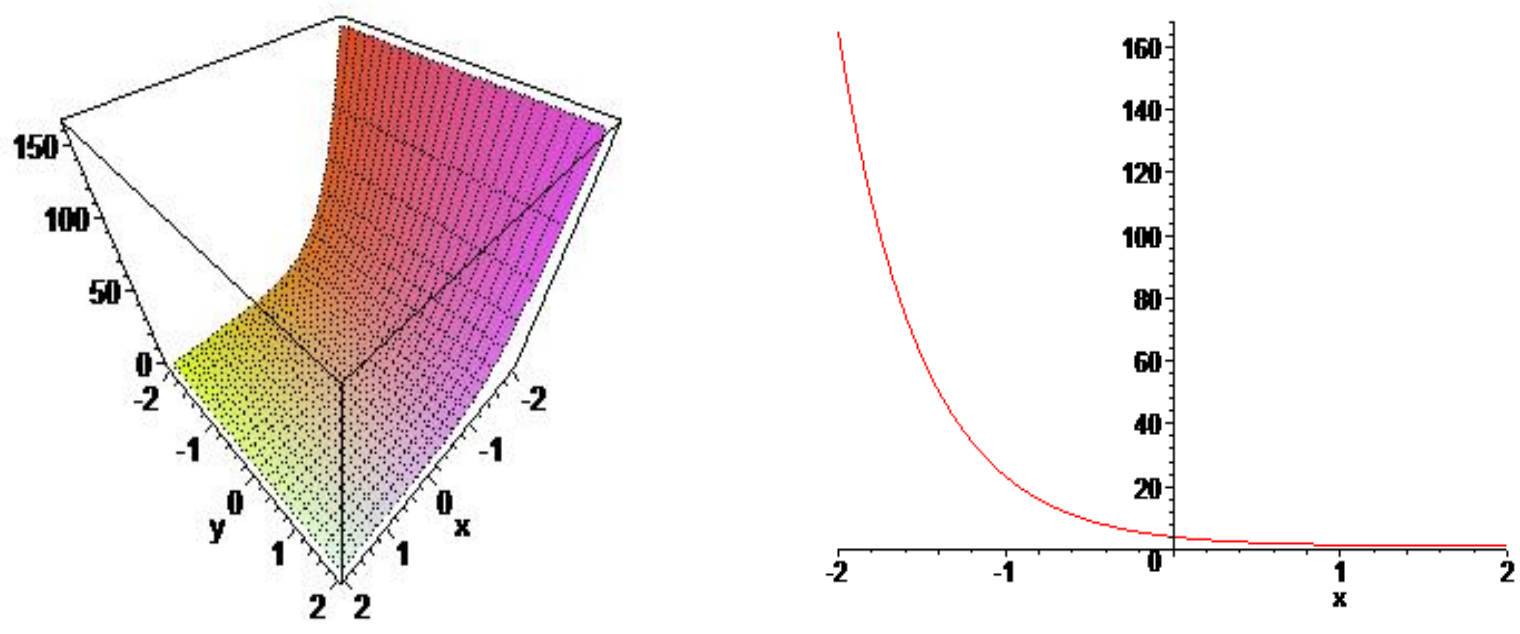

Fig. 2. 3D and 2D plots of travelling wave solutions (Case 2)

The plots indicate the wave solutions for $a_{-1}=3$ in Equation (16).

\section{Case 3:}

$$
\begin{gathered}
a_{0}=0, b_{0}=0, a_{1}=1+\sqrt{2}, a_{1}=1-\sqrt{2}, w=I \sqrt{2}, w=-I \sqrt{2} \\
a_{-1}=-(1+\sqrt{2}) b_{-1}+2 b_{-1}, a_{-1}=-(1-\sqrt{2}) b_{-1}+2 b_{-1}
\end{gathered}
$$

Replace these outcomes into Equation (6), we produce a subsequent exact solution,

$$
u(\xi)=\frac{\left(-(1+\sqrt{2}) b_{-1}+2 b_{-1}\right) e^{-\xi}+(1+\sqrt{2}) e^{\xi}}{b_{-1} e^{-\xi}+e^{\xi}}
$$

where $b_{-1}$ is a free parameter.
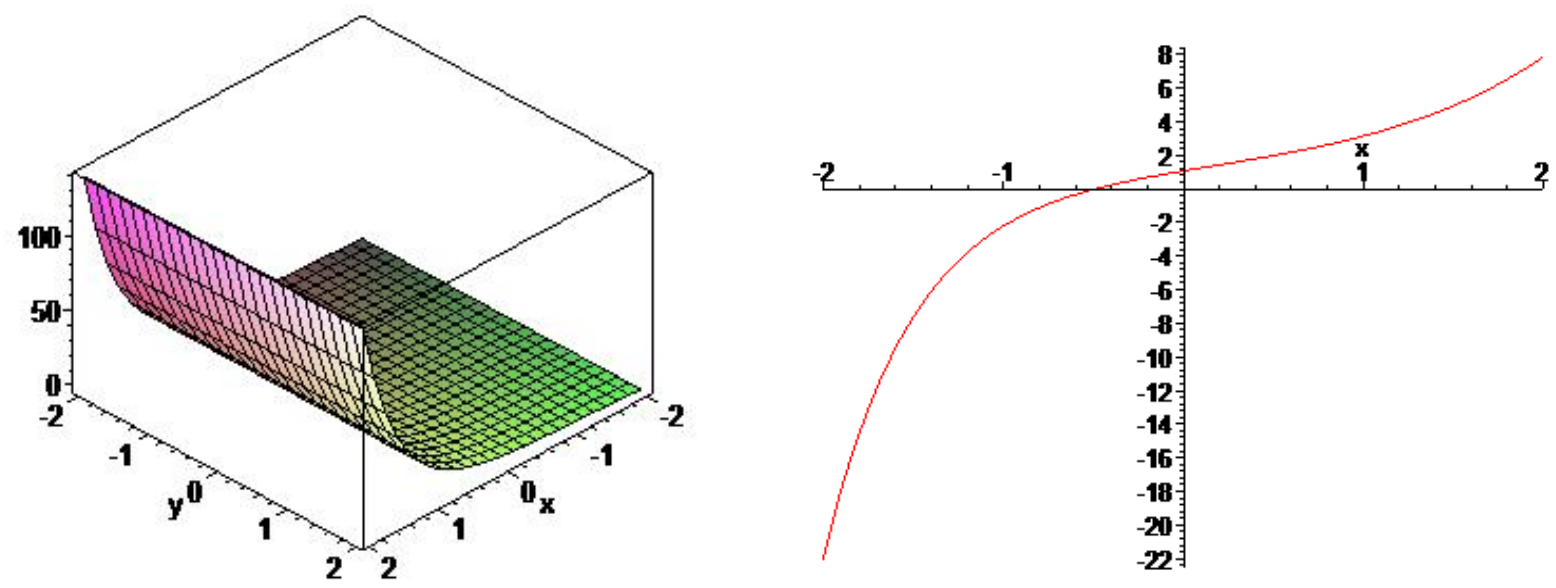

Fig. 3. 3D and 2D plots of travelling wave solutions (Case 3)

The plots indicate the wave solutions for $b_{-1}=5$ in Equation (18).

Case 4:

$$
a_{-1}=0, b_{-1}=0, a_{1}=1+\frac{1}{2} \sqrt{2}, a_{1}=1-\frac{1}{2} \sqrt{2}, w=\frac{1}{2} I \sqrt{14}
$$




$$
w=-\frac{1}{2} I \sqrt{14}, a_{0}=-\left(1-\frac{1}{2} \sqrt{2}\right) b_{0}+2 b_{0}, a_{0}=-\left(1+\frac{1}{2} \sqrt{2}\right) b_{0}+2 b_{0}
$$

Replace these outcomes into Equation (6), we produce a subsequent exact solution,

$$
u(\xi)=\frac{-\left(1-\frac{1}{2} \sqrt{2}\right) b_{0}+2 b_{0}+\left(1-\frac{1}{2} \sqrt{2}\right) e^{\xi}}{b_{0}+e^{\xi}}
$$

where $b_{0}$ is a free parameter.
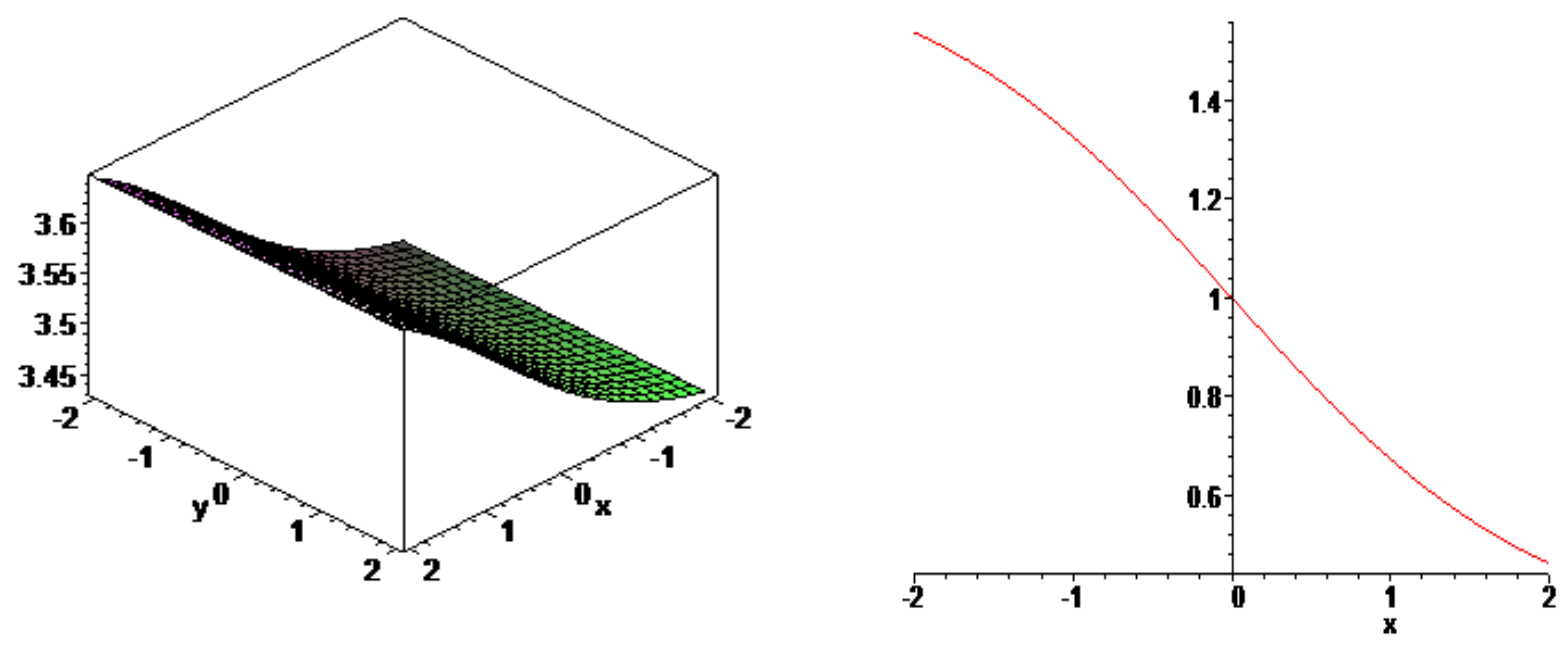

Fig. 4. 3D and 2D plots of travelling wave solutions (Case 4)

The plots indicate the wave solutions for $b_{0}=2$ in Equation (20).

\section{Case 5:}

$$
a_{-1}=0, b_{0}=a_{0}, a_{1}=0, w=I \sqrt{2}, w=-I \sqrt{2}, b_{-1}=\frac{1}{8} a_{0}{ }^{2}
$$

Replace these outcomes into Equation (6), we produce a subsequent exact solution,

$$
u(\xi)=\frac{a_{0}}{\frac{1}{8} a_{0}^{2} e^{-\xi}+a_{0}+e^{\xi}}
$$

where $a_{0}$ is a free parameter.
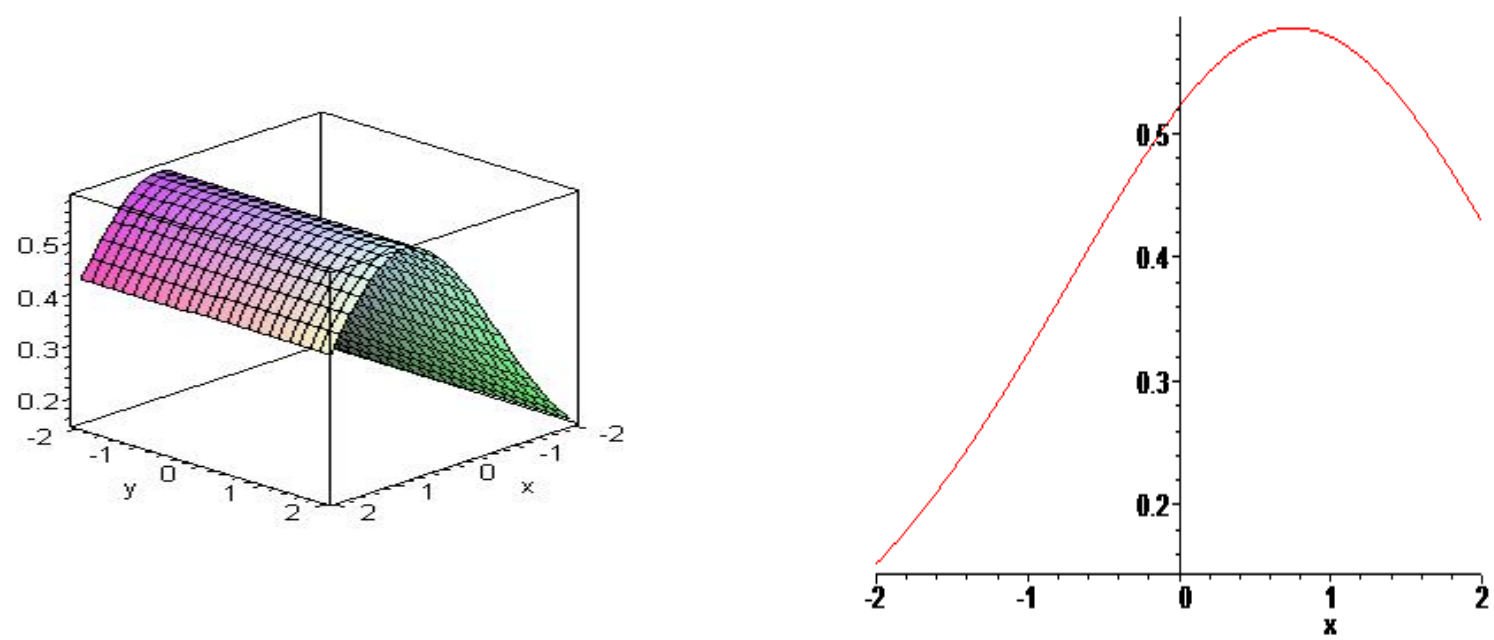

Fig. 5. 3D and 2D plots of travelling wave solutions (Case 5) 
The plots indicate the wave solutions for $a_{0}=6$ in Equation (22).

Case 6:

$$
\begin{gathered}
a_{-1}=-a_{0}{ }^{2}+\frac{1}{2}\left(1-\frac{1}{2} \sqrt{2}\right) a_{0}{ }^{2}-\left(1-\frac{1}{2} \sqrt{2}\right) b_{0} a_{0}+\frac{1}{4}\left(1-\frac{1}{2} \sqrt{2}\right) b_{0}{ }^{2}+2 b_{0} a_{0}-\frac{1}{2} b_{0}{ }^{2} \\
a_{-1}=-a_{0}{ }^{2}+\frac{1}{2}\left(1+\frac{1}{2} \sqrt{2}\right) a_{0}{ }^{2}-\left(1+\frac{1}{2} \sqrt{2}\right) b_{0} a_{0}+\frac{1}{4}\left(1+\frac{1}{2} \sqrt{2}\right) b_{0}{ }^{2}+2 b_{0} a_{0}-\frac{1}{2} b_{0}{ }^{2} \\
b_{-1}=b_{0} a_{0}-\frac{1}{2} a_{0}{ }^{2}-\frac{1}{4} b_{0}{ }^{2}, w=\frac{1}{2} I \sqrt{14}, w=-\frac{1}{2} I \sqrt{14}, a_{1}=1-\frac{1}{2} \sqrt{2}, a_{1}=1+\frac{1}{2} \sqrt{2}
\end{gathered}
$$

Replace these outcomes into Equation (6), we produce a subsequent exact solution,

$$
u(\xi)=\frac{\left(-a_{0}{ }^{2}+\frac{1}{2}\left(1-\frac{1}{2} \sqrt{2}\right) a_{0}{ }^{2}-\left(1-\frac{1}{2} \sqrt{2}\right) b_{0} a_{0}+\frac{1}{4}\left(1-\frac{1}{2} \sqrt{2}\right) b_{0}{ }^{2}+2 b_{0} a_{0}-\frac{1}{2} b_{0}{ }^{2}\right) e^{-\xi}+a_{0}+\left(1-\frac{1}{2} \sqrt{2}\right) e^{\xi}}{\left(b_{0} a_{0}-\frac{1}{2} a_{0}{ }^{2}-\frac{1}{4} b_{0}{ }^{2}\right) e^{-\xi}+b_{0}+e^{\xi}}
$$

where $b_{0}$ and $a_{0}$ are free parameters.
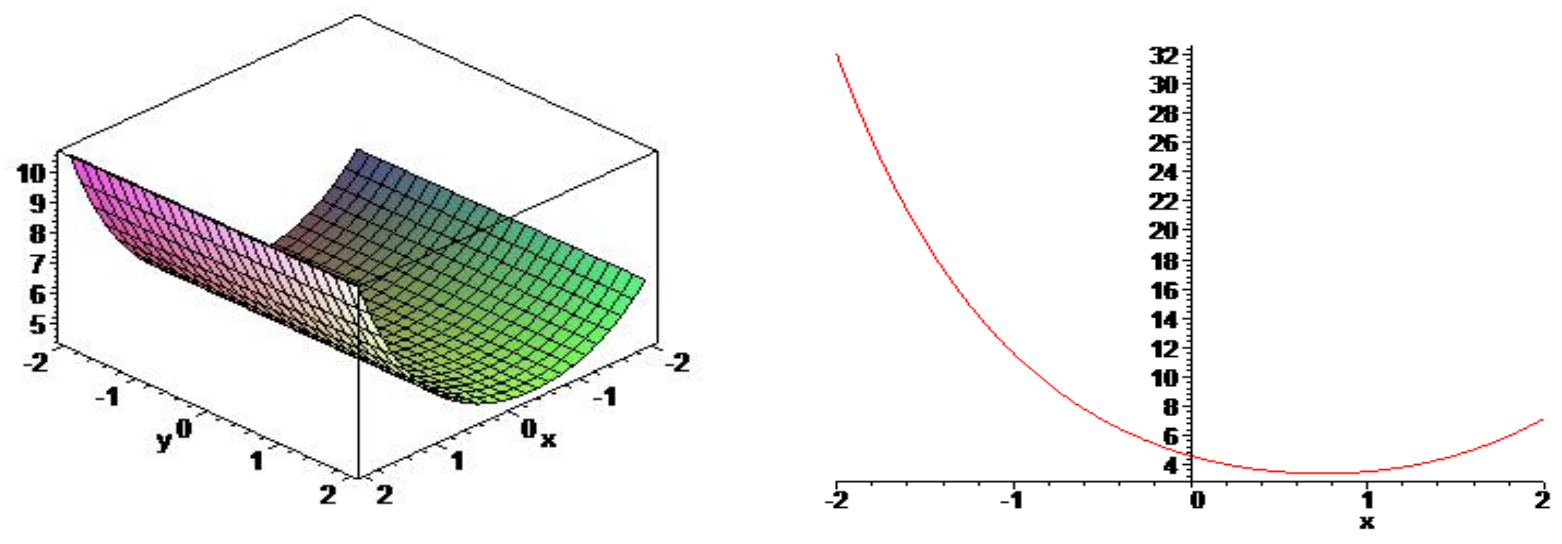

Fig. 6. 3D and 2D plots of travelling wave solutions (Case 6)

The plots indicate the wave solutions for $a_{0}=1, b_{0}=1$ in Equation (24).

Case 7:

$$
\begin{gathered}
a_{0}=\frac{b_{0}\left(a_{1}^{2}-a_{1}-1\right)}{-1+a_{1}}, w=\sqrt{-6 a_{1}+3 a_{1}^{2}-2}, w=-\sqrt{-6 a_{1}+3 a_{1}^{2}-2} \\
b_{-1}=\frac{1}{8} \frac{b_{0}^{2}\left(2 a_{1}{ }^{2}-4 a_{1}+1\right)}{\left(-1+a_{1}\right)^{2}}, a_{-1}=\frac{1}{8} \frac{b_{0}^{2}\left(2 a_{1}{ }^{2}-4 a_{1}+1\right) a_{1}}{\left(-1+a_{1}\right)^{2}}
\end{gathered}
$$

Replace these outcomes into Equation (6), we produce a subsequent exact solution,

$$
u(\xi)=\frac{\left(\frac{1}{8} \frac{b_{0}{ }^{2}\left(2 a_{1}{ }^{2}-4 a_{1}+1\right) a_{1}}{\left(-1+a_{1}\right)^{2}}\right) e^{-\xi}+\frac{b_{0}\left(a_{1}{ }^{2}-a_{1}-1\right)}{-1+a_{1}}+a_{1} e^{\xi}}{\left(\frac{1}{8} \frac{b_{0}{ }^{2}\left(2 a_{1}{ }^{2}-4 a_{1}+1\right)}{\left(-1+a_{1}\right)^{2}}\right) e^{-\xi}+b_{0}+e^{\xi}}
$$

where $b_{0}$ and $a_{1}$ are free parameters. 

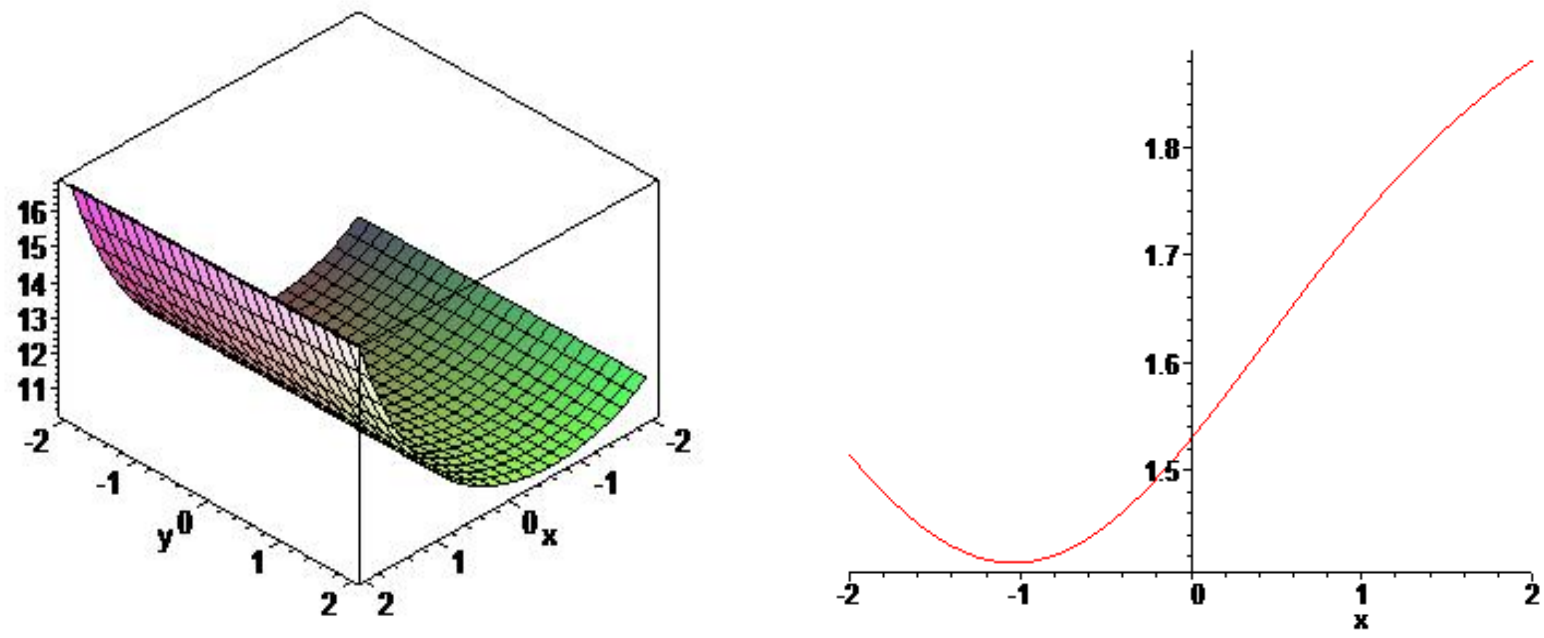

Fig. 7. 3D and 2D plots of travelling wave solutions (Case 7)

The plots indicate the wave solutions for $a_{1}=2, b_{0}=1$ in Equation (26).

Case 8:

$$
a_{-1}=-\frac{1}{8} a_{0}{ }^{2}, b_{0}=0, a_{1}=1, w=I \sqrt{5}, w=-I \sqrt{5}, b_{-1}=\frac{1}{8} a_{0}{ }^{2}
$$

Replace these outcomes into Equation (6), we produce a subsequent exact solution,

$$
u(\xi)=\frac{-a_{0}^{2} e^{-\xi}+8 a_{0}+8 e^{\xi}}{a_{0}^{2} e^{-\xi}+8 e^{\xi}}
$$

where $a_{0}$ is a free parameter.
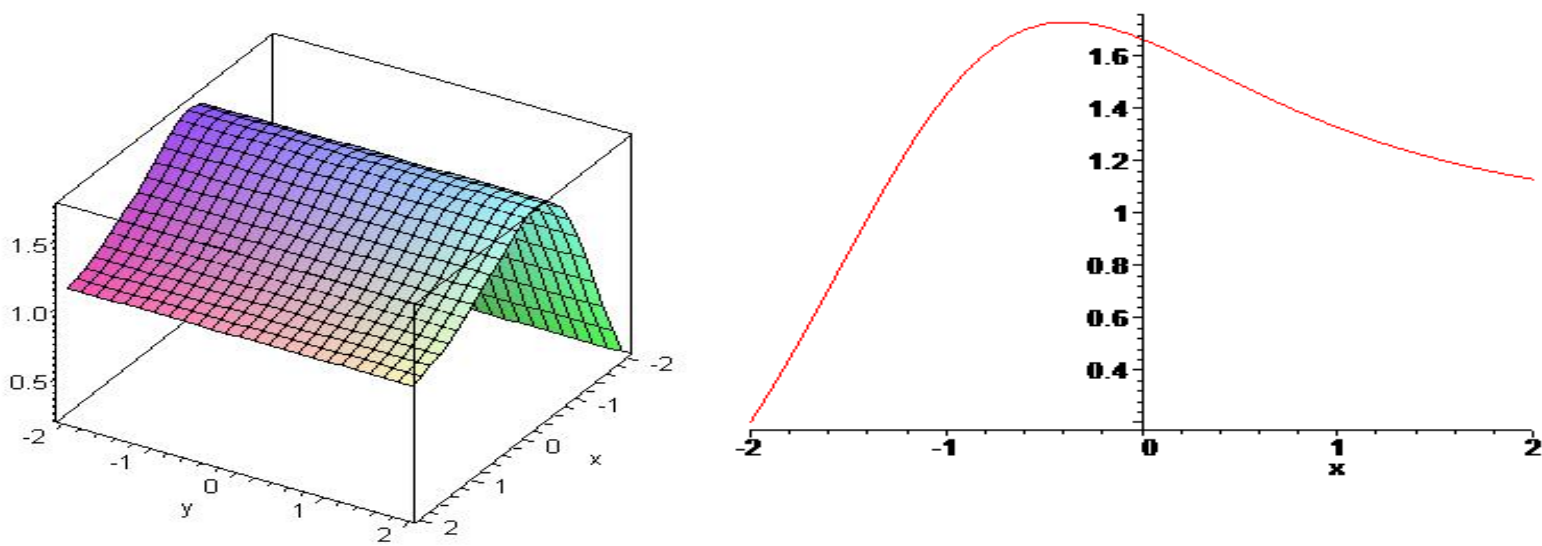

Fig. 8. 3D and 2D plots of travelling wave solutions (Case 8)

The plots indicate the wave solutions for $a_{0}=1$ in Equation (28).

Therefore, the full solutions of the Time-Fractional Bad Modified Boussinesq equation for the above conditions have been obtained. Now let's solve the Time-Fractional Good Modified Boussinesq equation using the Exp-function method.

We balance the linear term of the highest order of Equation (11) $u^{l v}$ with the highest order nonlinear term $u^{2} u^{\prime \prime}$, we set $p=c=1$ and $q=d=1$; then the trial solution, Equation (11), reduces to 


$$
\frac{-1}{B}\left[D_{4} e^{4 \xi}+D_{3} e^{3 \xi}+D_{2} e^{2 \xi}+D_{1} e^{\xi}+D_{0}+D_{-1} e^{-\xi}+D_{-2} e^{-2 \xi}+D_{-3} e^{-3 \xi}+D_{-4} e^{-4 \xi}\right]=0
$$

and for the solutions of Equation (29), all coefficients must be zero.

$$
\begin{aligned}
& B=\left(b_{-1} e^{-\xi}+b_{0}+e^{\xi}\right)^{5} \\
& D_{4}=w^{2} a_{1} b_{0}-3 a_{1}^{3} b_{0}-6 a_{1} a_{0}+6 a_{1}^{2} b_{0}-w^{2} a_{0}+3 a_{1}^{2} a_{0} \\
& D_{3}=24 a_{1}^{2} b_{-1}-12 a_{1} b_{-1}-24 a_{1} a_{-1}+12 a_{1}^{2} a_{-1}+12 a_{1} b_{0}^{2}+12 a_{1} a_{0}^{2}-12 a_{1}^{3} b_{-1}-4 w^{2} a_{1} \\
& +9 a_{1}^{3} b_{0}^{2}-21 a_{0} a_{1}^{2} b_{0}-6 a_{1}^{2} b_{0}^{2}-12 a_{0} b_{0}+4 w^{2} a_{1} b_{-1}-w^{2} a_{0} b_{0}+w^{2} a_{1} b_{0}^{2}-12 a_{0}^{2}+12 a_{-1} \\
& +18 a_{1} b_{0} a_{0} \text {; } \\
& D_{2}=-12 a_{1} b_{0}^{3}-72 a_{0} b_{-1}-12 a_{1}^{2} b_{0}^{3}-12 a_{-1} b_{0}-66 a_{1}^{2} b_{-1} a_{0}+12 a_{0} b_{0}^{2}-54 a_{-1} a_{0}+9 a_{0}^{3} \\
& +18 a_{1} b_{0}^{2} a_{0}-11 w^{2} a_{-1} b_{0}+33 a_{1}^{3} b_{-1} b_{0}+12 a_{0} a_{1}^{2} b_{0}^{2}-21 a_{1}^{2} b_{-1} b_{0}+54 a_{-1} a_{0} a_{1}-12 a_{-1} a_{1} b_{0} \\
& +84 a_{1} b_{-1} b_{0}+4 w^{2} a_{0} b_{-1}+w^{2} a_{0} b_{0}^{2}-21 a_{1} b_{0} a_{0}^{2}-w^{2} a_{1} b_{0}^{3}+78 a_{1} b_{-1} a_{0}-12 a_{1}^{2} b_{-1} b_{0} \\
& -6 a_{0}^{2} b_{0}+7 w^{2} a_{1} b_{-1} b_{0} \\
& D_{1}=-3 a_{0}^{3} b_{0}+180 a_{1} b_{-1}^{2}+36 a_{0}^{2} b_{-1}+48 a_{-1} a_{0}^{2}-24 a_{1}^{2} b_{-1}^{2}+6 a_{0}^{2} b_{0}^{2}+48 a_{1} a_{-1}^{2}+36 a_{1}^{3} b_{-1}^{2} \\
& -180 a_{-1} b_{-1}+3 a_{-1} a_{1}^{2} b_{0}^{2}-18 a_{-1} a_{0} a_{1} b_{0}-2 w^{2} a_{1} b_{0}^{2} b_{-1}-11 w^{2} a_{-1} b_{0}^{2}-78 a_{-1} a_{0} b_{0} \\
& +13 w^{2} a_{0} b_{0} b_{-1}+84 a_{1} b_{-1} a_{0} b_{0}+51 a_{0} a_{1}^{2} b_{-1} b_{0}+72 a_{-1} a_{1} b_{-1}-84 a_{1} b_{-1} a_{0}^{2}-54 a_{1}^{2} b_{-1} b_{0}^{2} \\
& +60 a_{0} b_{-1} b_{0}+4 w^{2} a_{1} b_{-1}^{2}-6 a_{0} a_{1} b_{0}^{3}-48 a_{-1}^{2}+w^{2} a_{0} b_{0}^{3}+12 a_{-1} a_{1} b_{0}^{2}-84 a_{1}^{2} a_{-1} b_{-1} \\
& +3 a_{0}^{2} a_{1} b_{0}^{2}-w^{2} a_{1} b_{0}^{4}-60 a_{1} b_{-1} b_{0}^{2}-4 w^{2} a_{-1} b_{-1} ; \\
& D_{0}=-30 a_{0} a_{1} b_{-1}^{2}+75 a_{0} a_{1}^{2} b_{-1}^{2}+10 w^{2} a_{0} b_{-1}^{2}+240 a_{0} b_{-1}^{2}+120 a_{-1} a_{1} b_{-1} b_{0}+15 a_{1}^{2} a_{-1} b_{-1} b_{0} \\
& -90 a_{1}^{2} b_{-1}^{2} b_{0}-90 a_{-1}^{2} b_{0}-30 a_{-1}^{2} a_{0} b_{0}^{2}+15 a_{-1} b_{0} a_{0}^{2}-120 a_{1} b_{-1}^{2} b_{0}-120 a_{-1} b_{-1} b_{0} \\
& -30 a_{1} b_{0}^{2} a_{0} b_{-1}+15 a_{1} a_{-1}^{2} b_{0}-180 a_{0} a_{-1} a_{1} b_{-1}+10 w^{2} a_{0} b_{0}^{2} b_{-1}+15 a_{1} b_{0} a_{0}^{2} b_{-1}+75 a_{0} a_{-1}^{2} \\
& -5 w^{2} a_{-1} b_{-1} b_{0}-5 w^{2} a_{1} b_{0}^{3} b_{-1}-5 w^{2} a_{1} b_{0} b_{-1}^{2}-5 w^{2} a_{-1} b_{0}^{3}-30 a_{0}^{3} b_{-1}+60 a_{0}^{2} b_{-1} b_{0} \\
& +30 a_{0} a_{-1} b_{-1} \\
& D_{-1}=36 a_{-1}^{3}-6 a_{0} a_{-1} b_{0}^{3}-4 w^{2} a_{1} b_{-1}^{3}-3 a_{0}^{3} b_{-1} b_{0}+3 a_{0}^{2} a_{-1} b_{0}^{2}+6 a_{0}^{2} b_{-1} b_{0}^{2}-w^{2} a_{-1} b_{0}^{4} \\
& -84 a_{-1}^{2} a_{1} b_{-1}-84 a_{0}^{2} a_{-1} b_{-1}+48 a_{1} a_{0}^{2} b_{-1}^{2}+60 a_{0} b_{-1}^{2} b_{0}-60 a_{-1} b_{-1} b_{0}^{2}+3 a_{-1}^{2} a_{1} b_{0}^{2} \\
& +4 w^{2} a_{-1} b_{-1}^{2}+51 a_{0} a_{-1}^{2} b_{0}+48 a_{-1} a_{1}^{2} b_{-1}^{2}+72 a_{-1} a_{1} b_{-1}^{2}+180 a_{-1} b_{-1}^{2}-180 a_{1} b_{-1}^{3}-48 a_{1}^{2} b_{-1}^{3} \\
& -54 a_{-1}^{2} b_{0}^{2}-24 a_{-1}^{2} b_{-1}+36 a_{0}^{2} b_{-1}^{2}+w^{2} a_{0} b_{-1} b_{0}^{3}+13 w^{2} a_{0} b_{-1}^{2} b_{0}-78 a_{1} b_{-1}^{2} a_{0} b_{0} \\
& -11 w^{2} a_{1} b_{0}^{2} b_{-1}^{2}-18 a_{0} a_{-1} a_{1} b_{0} b_{-1}+12 a_{-1} b_{0}^{2} a_{1} b_{-1}-2 w^{2} a_{-1} b_{0}^{2} b_{-1}+84 a_{-1} a_{0} b_{0} b_{-1} \text {; } \\
& D_{-2}=w^{2} a_{0} b_{-1}^{2} b_{0}^{2}+54 a_{-1} a_{0} a_{1} b_{-1}^{2}-21 a_{-1} a_{0}^{2} b_{0} b_{-1}-11 w^{2} a_{1} b_{-1}^{3} b_{0}+18 a_{-1} b_{0}^{2} a_{0} b_{-1}
\end{aligned}
$$




$$
\begin{aligned}
& +7 w^{2} a_{-1} b_{-1}^{2} b_{0}-12 a_{-1} b_{0} a_{1} b_{-1}^{2}-21 a_{-1}^{2} a_{1} b_{0} b_{-1}-w^{2} a_{-1} b_{0}^{3} b_{-1}+9 a_{0}^{3} b_{-1}^{2}-72 a_{0} b_{-1}^{3} \\
& +33 a_{-1}^{3} b_{0}-12 a_{-1}^{2} b_{0}^{3}-66 a_{-1}^{2} a_{0} b_{-1}+84 a_{-1} b_{-1}^{2} b_{0}-12 a_{1} b_{-1}^{3} b_{0}+78 a_{-1} a_{0} b_{-1}^{2}-6 a_{0}^{2} b_{-1}^{2} b_{0} \\
& +4 w^{2} a_{0} b_{-1}^{3}-12 a_{-1} b_{-1} b_{0}^{3}+12 a_{0} b_{-1}^{2} b_{0}^{2}+12 a_{0} a_{-1}^{2} b_{0}^{2}-54 a_{1} b_{-1}^{3} a_{0}-12 a_{-1}^{2} b_{0} b_{-1} ; \\
D_{-3}= & 24 a_{-1}^{2} b_{-1}^{2}+12 a_{1} b_{-1}^{4}-12 a_{-1} b_{-1}^{3}+9 a_{-1}^{3} b_{0}^{2}-12 a_{0}^{2} b_{-1}^{3}-12 a_{-1}^{3} b_{-1}+12 a_{-1} a_{0}^{2} b_{-1}^{2} \\
& +12 a_{-1} b_{-1}^{2} b_{0}^{2}-12 a_{0} b_{-1}^{3} b_{0}+w^{2} a_{-1} b_{-1}^{2} b_{0}^{2}-21 a_{-1}^{2} a_{0} b_{-1} b_{0}+18 a_{-1} a_{0} b_{-1}^{2} b_{0}-w^{2} a_{0} b_{-1}^{3} b_{0} \\
& +12 a_{-1}^{2} a_{1} b_{-1}^{2}-4 w^{2} a_{1} b_{-1}^{4}-6 a_{-1}^{2} b_{0}^{2} b_{-1}+4 w^{2} a_{-1} b_{-1}^{3}-24 a_{-1} a_{1} b_{-1}^{3} ; \\
D_{-4} & =w^{2} a_{-1} b_{-1}^{3} b_{0}-3 a_{-1}^{3} b_{-1} b_{0}-w^{2} a_{0} b_{-1}^{4}-6 a_{-1} a_{0} b_{-1}^{3}+6 a_{-1}^{2} b_{-1}^{2} b_{0}+3 a_{-1}^{2} a_{0} b_{-1}^{2} ;
\end{aligned}
$$

All the coefficients of $e^{n \xi}$ must be zero. Hence, we produce a system of algebraic equations which the Maple can tackle to produce the subsequent cases of solutions:

\section{Case 1:}

$$
a_{0}=a_{1} b_{0}, a_{-1}=a_{1} b_{-1}
$$

Replace these outcomes into Equation (6), we produce a subsequent exact solution,

$$
u(\xi)=a_{1}
$$

where $a_{1}$ is a free parameter.
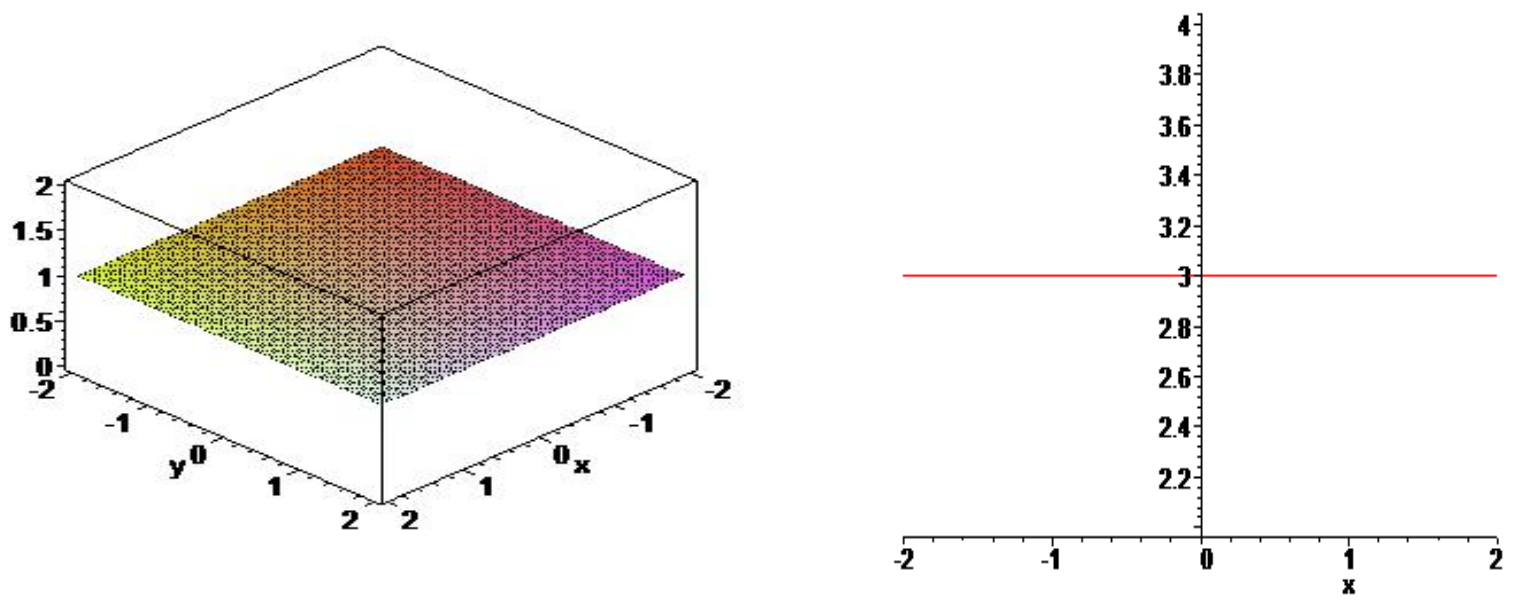

Fig. 9. 3D and 2D plots of travelling wave solutions (Case 1)

The plots indicate the wave solutions for $a_{1}=3$ in Equation (31).

Case 2:

$$
a_{0}=0, b_{0}=0, a_{1}=1, w=0, b_{-1}=0
$$

Replace these outcomes into Equation (6), we produce a subsequent exact solution,

$$
u(\xi)=\frac{a_{-1} e^{-\xi}+e^{\xi}}{e^{\xi}}
$$


where $a_{-1}$ is a free parameter.
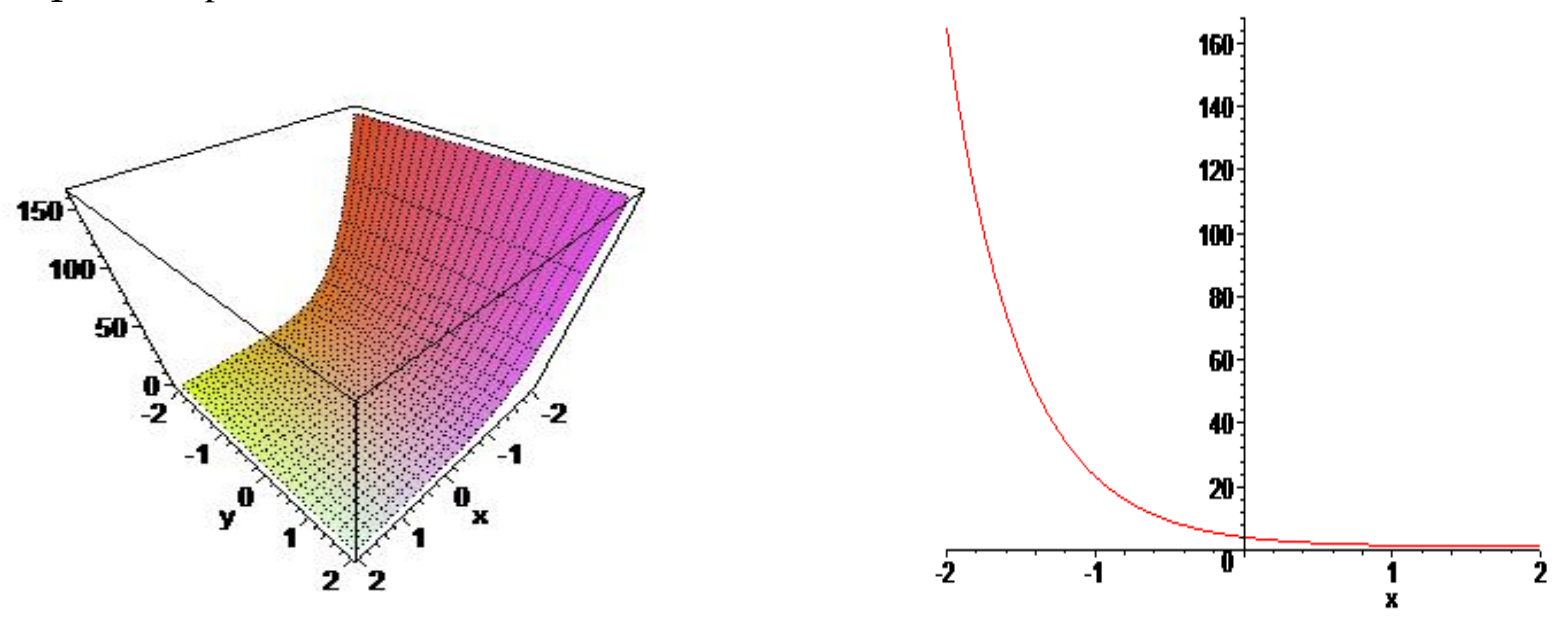

Fig. 10. 3D and 2D plots of travelling wave solutions (Case 2)

The plots indicate the wave solutions for $a_{-1}=3$ in Equation (33).

Case 3:

$$
\begin{gathered}
a_{0}=0, b_{0}=0, a_{1}=1+I \sqrt{2}, a_{1}=1-I \sqrt{2}, w=I \sqrt{6}, w=-I \sqrt{6} \\
a_{-1}=-(1+I \sqrt{2}) b_{-1}+2 b_{-1}, a_{-1}=-(1-I \sqrt{2}) b_{-1}+2 b_{-1}
\end{gathered}
$$

Replace these outcomes into Equation (6), we produce a subsequent exact solution,

$$
u(\xi)=\frac{\left(-(1+I \sqrt{2}) b_{-1}+2 b_{-1}\right) e^{-\xi}+(1+I \sqrt{2}) e^{\xi}}{b_{-1} e^{-\xi}+e^{\xi}}
$$

where $b_{-1}$ is a free parameter.
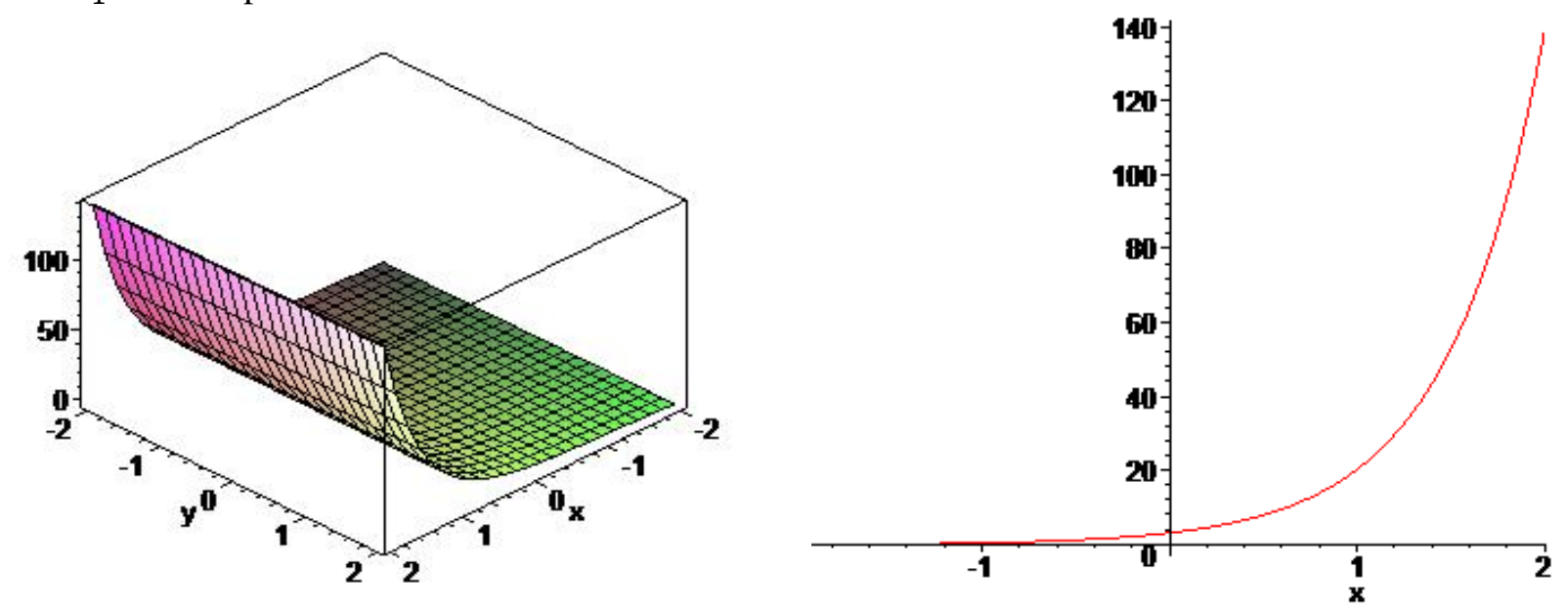

Fig. 11. 3D and 2D plots of travelling wave solutions (Case 3)

The plots indicate the wave solutions for $b_{-1}=1$ in Equation (35).

Case 4:

$$
\begin{gathered}
a_{-1}=0, b_{-1}=0, \quad a_{1}=1+\frac{1}{2} I \sqrt{2}, a_{1}=1-\frac{1}{2} I \sqrt{2}, w=\frac{3}{2} I \sqrt{2} \\
w=-\frac{3}{2} I \sqrt{2}, a_{0}=-\left(1-\frac{1}{2} I \sqrt{2}\right) b_{0}+2 b_{0}, a_{0}=-\left(1+\frac{1}{2} I \sqrt{2}\right) b_{0}+2 b_{0}
\end{gathered}
$$


Replace these outcomes into Equation (6), we produce a subsequent exact solution,

$$
u(\xi)=\frac{-\left(1-\frac{1}{2} \sqrt{2}\right) b_{0}+2 b_{0}+\left(1-\frac{1}{2} \sqrt{2}\right) e^{\xi}}{b_{0}+e^{\xi}}
$$

where $b_{0}$ is a free parameter.
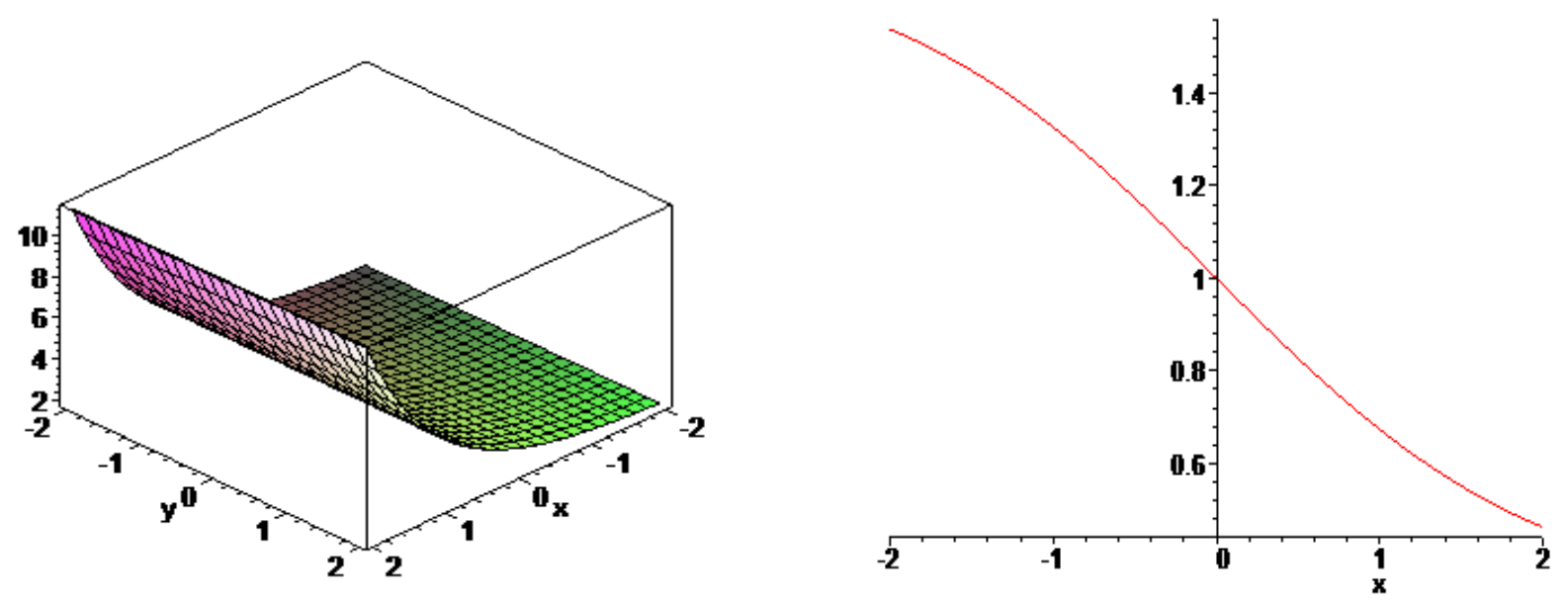

Fig. 12. 3D and 2D plots of travelling wave solutions (Case 4)

The plots indicate the wave solutions for $b_{0}=1$ in Equation (37).

\section{Case 5:}

$$
a_{-1}=0, b_{0}=-a_{0}, a_{1}=0, w=0, b_{-1}=\frac{3}{8} a_{0}^{2}
$$

Replace these outcomes into Equation (6), we produce a subsequent exact solution,

$$
u(\xi)=\frac{a_{0}}{\frac{3}{8} a_{0}^{2} e^{-\xi}-a_{0}+e^{\xi}}
$$

where $a_{0}$ is a free parameter.
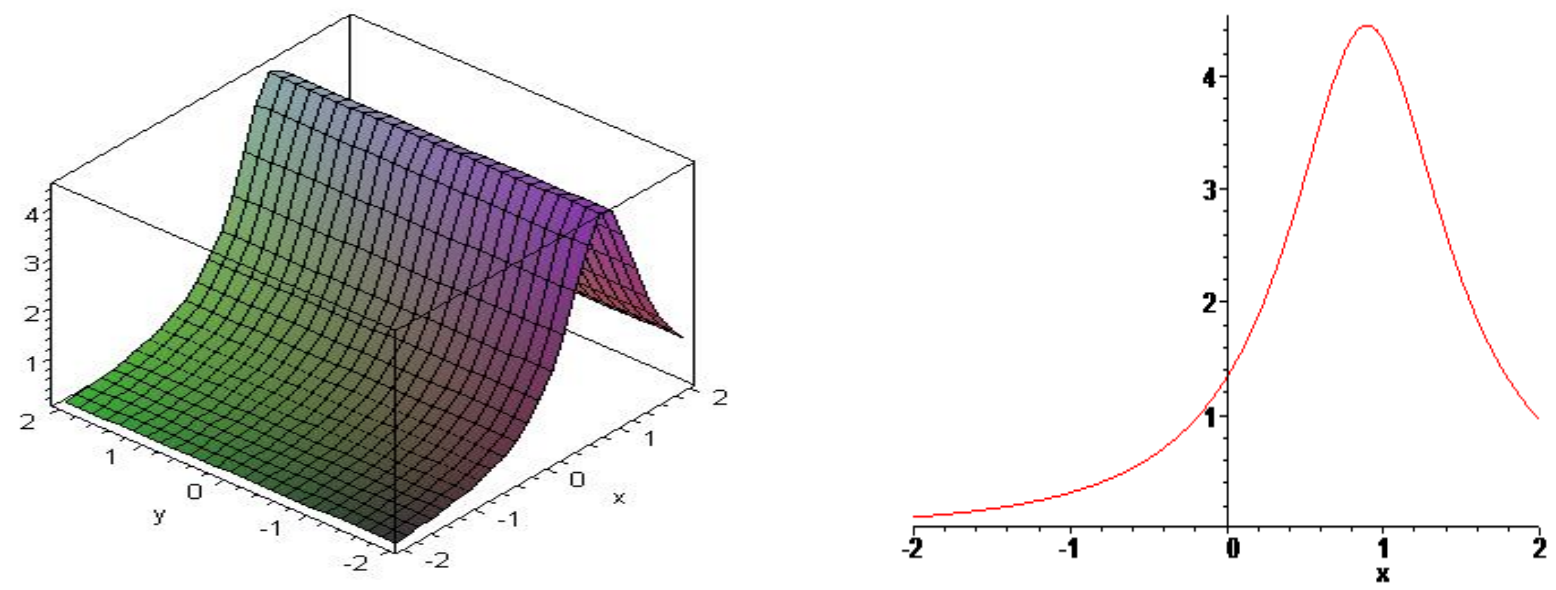

Fig. 13. 3D and 2D plots of travelling wave solutions (Case 5) 
The plots indicate the wave solutions for $a_{0}=4$ in Equation (39).

Case 6:

$$
\begin{gathered}
a_{-1}=a_{0}{ }^{2}-\frac{1}{2}\left(1+\frac{1}{2} I \sqrt{2}\right) a_{0}{ }^{2}+\left(1+\frac{1}{2} I \sqrt{2}\right) b_{0} a_{0}-\frac{3}{4}\left(1+\frac{1}{2} I \sqrt{2}\right) b_{0}{ }^{2}-2 b_{0} a_{0}+\frac{3}{2} b_{0}{ }^{2} \\
a_{-1}=a_{0}{ }^{2}-\frac{1}{2}\left(1-\frac{1}{2} I \sqrt{2}\right) a_{0}{ }^{2}+\left(1-\frac{1}{2} I \sqrt{2}\right) b_{0} a_{0}-\frac{3}{4}\left(1-\frac{1}{2} I \sqrt{2}\right) b_{0}{ }^{2}-2 b_{0} a_{0}+\frac{3}{2} b_{0}{ }^{2} \\
b_{-1}=-b_{0} a_{0}+\frac{1}{2} a_{0}{ }^{2}+\frac{3}{4} b_{0}{ }^{2}, w=\frac{3}{2} I \sqrt{2}, w=-\frac{3}{2} I \sqrt{2}, a_{1}=1-\frac{1}{2} I \sqrt{2}, a_{1}=1+\frac{1}{2} I \sqrt{2}
\end{gathered}
$$

Replace these outcomes into Equation (6), we produce a subsequent exact solution,

$$
u(\xi)=\frac{\left(a_{0}{ }^{2}-\frac{1}{2}\left(1+\frac{1}{2} I \sqrt{2}\right) a_{0}{ }^{2}+\left(1+\frac{1}{2} I \sqrt{2}\right) b_{0} a_{0}-\frac{3}{4}\left(1+\frac{1}{2} I \sqrt{2}\right) b_{0}{ }^{2}-2 b_{0} a_{0}+\frac{3}{2} b_{0}{ }^{2}\right) e^{-\xi}+a_{0}+\left(1-\frac{1}{2} I \sqrt{2}\right) e^{\xi}}{\left(-b_{0} a_{0}+\frac{1}{2} a_{0}{ }^{2}+\frac{3}{4} b_{0}{ }^{2}\right) e^{-\xi}+b_{0}+e^{\xi}}
$$

where $b_{0}$ and $a_{0}$ are free parameters.
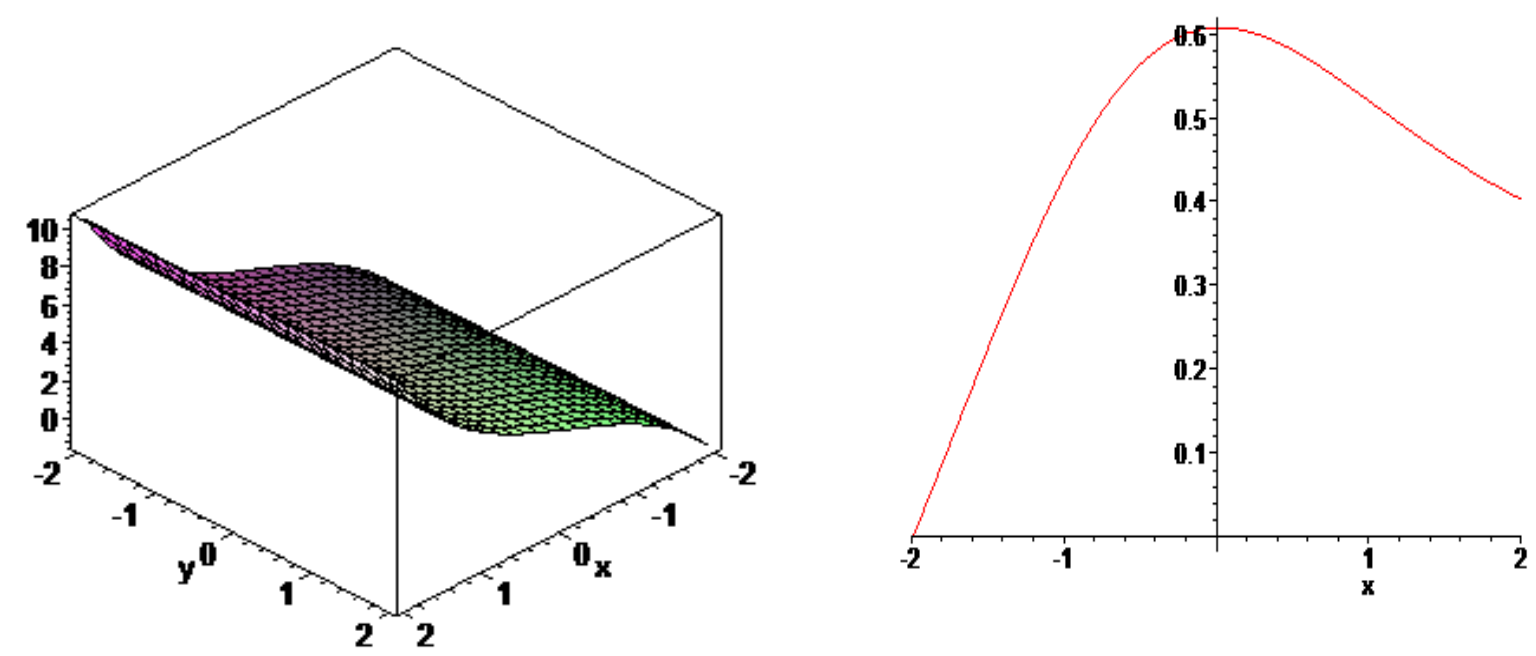

Fig. 14. 3D and 2D plots of travelling wave solutions (Case 6)

The plots indicate the wave solutions for $a_{0}=1, b_{0}=1$ in Equation (41)

Case 7:

$$
\begin{gathered}
a_{0}=\frac{b_{0}\left(a_{1}{ }^{2}-a_{1}+1\right)}{-1+a_{1}}, w=\sqrt{-6 a_{1}+3 a_{1}^{2}}, w=-\sqrt{-6 a_{1}+3 a_{1}^{2}} \\
b_{-1}=\frac{1}{8} \frac{b_{0}{ }^{2}\left(2 a_{1}{ }^{2}-4 a_{1}+3\right)}{\left(-1+a_{1}\right)^{2}}, a_{-1}=\frac{1}{8} \frac{b_{0}{ }^{2}\left(2 a_{1}{ }^{2}-4 a_{1}+3\right) a_{1}}{\left(-1+a_{1}\right)^{2}}
\end{gathered}
$$

Replace these outcomes into Equation (6), we produce a subsequent exact solution,

$$
u(\xi)=\frac{\left(\frac{1}{8} \frac{b_{0}{ }^{2}\left(2 a_{1}{ }^{2}-4 a_{1}+3\right) a_{1}}{\left(-1+a_{1}\right)^{2}}\right) e^{-\xi}+\frac{b_{0}\left(a_{1}{ }^{2}-a_{1}+1\right)}{-1+a_{1}}+a_{1} e^{\xi}}{\left(\frac{1}{8} \frac{b_{0}{ }^{2}\left(2 a_{1}{ }^{2}-4 a_{1}+3\right)}{\left(-1+a_{1}\right)^{2}}\right) e^{-\xi}+b_{0}+e^{\xi}}
$$

where $b_{0}$ and $a_{1}$ are free parameters. 

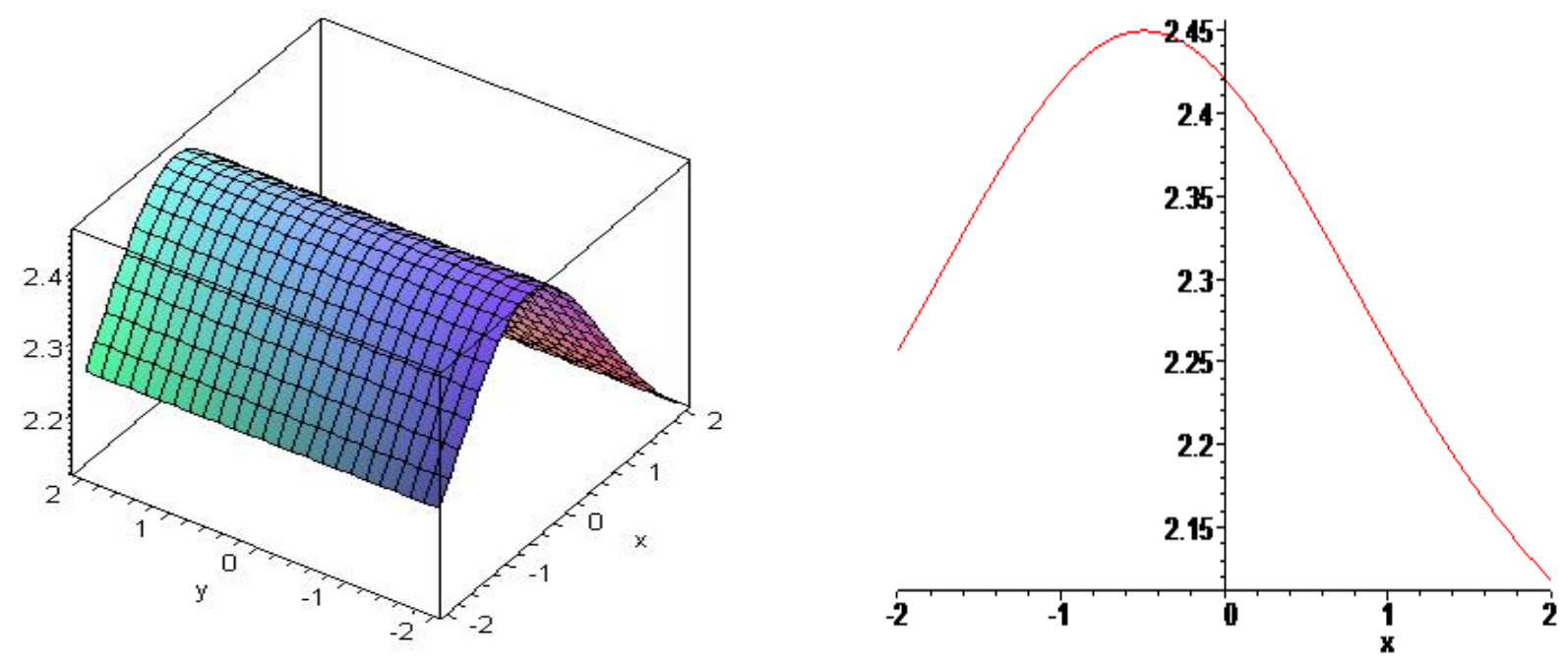

Fig.15. 3D and 2D plots of travelling wave solutions (Case 7)

The plots indicate the wave solutions for $a_{1}=2, b_{0}=1$ in Equation (43).

\section{Case 8:}

$$
a_{-1}=\frac{1}{8} a_{0}^{2}, b_{0}=0, \quad a_{1}=1, w=I \sqrt{3}, w=-I \sqrt{3}, b_{-1}=\frac{1}{8} a_{0}^{2}
$$

Replace these outcomes into Equation (6), we produce a subsequent exact solution,

$$
u(\xi)=\frac{-a_{0}^{2} e^{-\xi}+8 a_{0}+8 e^{\xi}}{a_{0}^{2} e^{-\xi}+8 e^{\xi}}
$$

where $a_{0}$ is a free parameter.
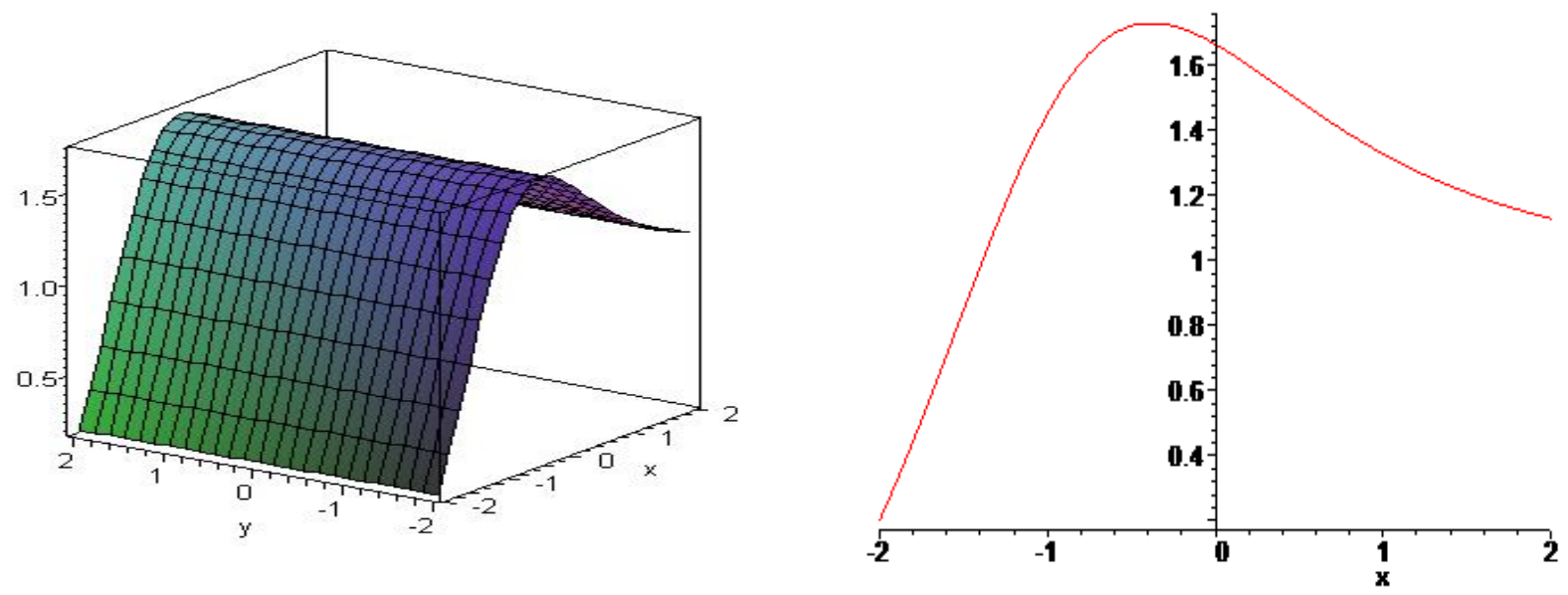

Fig. 16. 3D and 2D plots of travelling wave solutions (Case 8)

The plots indicate the wave solutions for $a_{0}=1$ in Equation (45).

Remark: With the aid of Maple, we have verified all solutions in Section 3 by putting them back into the originals Equations (10) and (11). 


\section{Conclusion}

In this paper, we have been obtained the new exact solution of the Conformable Time Fractional Bad and Good Modified Boussinesq Equations. We converted the Conformable Time Fractional Bad and Good Modified Boussinesq Equations into an ordinary differential equation with the help of a travelling wave transformation. We obtained new exact solutions by using the Exp-function method, which is different from previous literature works. These results show that the Exp-function method is a powerful and effective method to obtain the exact solutions of nonlinear evolution equations born in mathematical physics and non-linear dynamic systems.

\section{Author Contributions}

All the authors contributed equally to this work. They all read and approved the last version of the manuscript.

\section{Conflict of Interest}

The authors declare no conflict of interest.

\section{References}

[1] J. H. He, X. H. Wu, Exp-Function Method for Nonlinear Wave Equations, Chaos, Soliton and Fractals 30(3) (2006) 700-708.

[2] E. M. E. Zayed, M. A. M. Abdelaziz, The Tanh- Function Method Using a Generalized Wave Transformation for Nonlinear Equations, International Journal of Nonlinear Sciences and Numerical Simulation 11(8) (2010) 595-601.

[3] E. Fan, H. Zhang, A Note on the Homogeneous Balance Method, Physics Letters A 246(5) (1998) 403406.

[4] Y. Gurefe, A. Sönmezoğlu, E. Mrsırl1, Application of The Trial Equation Method for Solving Some Nonlinear Evolution Equations Arising in Mathematical Physics, Pramana 77(6) (2011) 1023-1029.

[5] N. A. Kudryashov, A Note on the $\left(\frac{G^{\prime}}{G}\right)$-expansion Method, Applied Mathematics and Computation 217(4) (2010) 1755-1758.

[6] P. N. Ryabov, D. I. Sinelshchikov, M. B. Kochanov, Application of the Kudryashov Method for Finding Exact Solutions of the High Order Nonlinear Evolution Equations, Applied Mathematics and Computation 218(7) (2011) 3965-3972.

[7] J. H. He, Exp-function Method for Fractional Differential Equations, International Journal of Nonlinear Sciences and Numerical Simulation 14(6) (2013) 363-366.

[8] Z. Öztürk, H. Bilgil, Exact Solutions of Rosenzweig-Macarthur (RM) Model Equations by Using Exp Function Method, European Journal of Science and Technology (16) (2019) 427-433.

[9] A. Ebaid, Application of the Exp-Function Method for Solving Some Evolution Equations with Nonlinear Terms of Any Orders, Zeitschrift fur Naturforschung A 65(12) (2010) 1039-1044.

[10] S. Zhang, Exp-Function Method for Riccati Equation and New Exact Solutions with Two Arbitrary Functions of $(2+1)$-Dimensional Konopelchenko-Dubrovsky Equations, Applied Mathematics and Computation 216(5) (2010) 1546-1552.

[11] R. Khalil, M. A. Horani, A. Yousef, M. Sababheh, A New Definition of Fractional Derivative, Journal of Computational and Applied Mathematics 264 (2014) 65-70. 
[12] I. Podlubny, Fractional Differential Equations: An Introduction to Fractional Derivatives, Fractional Differential Equations, to Methods of Their Solution and Some of Their Applications, Elsevier, 1998.

[13] A. Atangana, I. Koca, Chaos in A Simple Nonlinear System with Atangana-Baleanu Derivatives with Fractional Order, Chaos, Solitons and Fractals 89 (2016) 447-454.

[14] N. S. Kottakkaran, O. I Ilhan, S. T. Abdulazeez, J. Manafian, S. A. Mohammed, M. S. Osman, Novel Multiple Soliton Solutions for Some Nonlinear Pdes via Multiple Exp-Function Method, Results in Physics 21 (2021) 103769.

[15] K. A. Gepreel, E. M. E. Zayed, Multiple Wave Solutions for Nonlinear Burgers Equations Using the Multiple Exp-Function Method, International Journal of Modern Physics C (2021) 2150149.

[16] H. M. Ahmed, M. M. A. El-Sheikh, A. H. Arnous, W. B. Rabiel, Solitons and Other Solutions to $(N+1)$ Dimensional Modified Zakharov-Kuznetsov Equation by Exp-Function Method, SeMA Journal 78 (2021) $1-13$.

[17] A. G. Hossam, H. Abd-Allah, M. Zakarya, Exact Solutions of Stochastic Fractional Korteweg De-Vries Equation with Conformable Derivatives, Chinese Physics B 29(3) (2020) 30203.

[18] H. C. Yaslan, A. Girgin, Exp-Function Method for The Conformable Space-Time Fractional STO, ZKBBM and Coupled Boussinesq Equations, Arab Journal of Basic and Applied Sciences 26(1) (2019) $163-170$.

[19] L. K. Ravi, S. S. Ray, S. Sahoo, New Exact Solutions of Coupled Boussinesq-Burgers Equations by ExpFunction Method, Journal of Ocean Engineering and Science 2(1) (2017) 34-46.

[20] E. M. Eskandari, N. Taghizadeh, Exact Solutions of Two Nonlinear Space-time Fractional Differential Equations by Application of Exp-function Method, Applications and Applied Mathematics: An International Journal 15(2) (2020) 970-977.

[21] H. Durur, O. Taşbozan, A. Kurt, New Analytical Solutions of Conformable Time Fractional Bad and Good Modified Boussinesq Equations, Applied Mathematics and Nonlinear Sciences 5(1) (2020) 447454.

[22] A. Ebaid, An Improvement on The Exp-Function Method When Balancing the Highest Order Linear and Nonlinear Terms, Journal of Mathematical Analysis and Applications 392(1) (2012) 1-5.

[23] J. Boussinesq, Thorie Des Ondes Et Des Remous Qui Se Propagent Le Long D'un Canal Rectangulaire Horizantal,En Communiquant Au Liquide Contenu Dans Ce Canal Des Vitesses Sensiblement Pareilles De La Surface Au Fond, Journal de Mathmmatiques Pures at Appliques (1872) 55-108. 